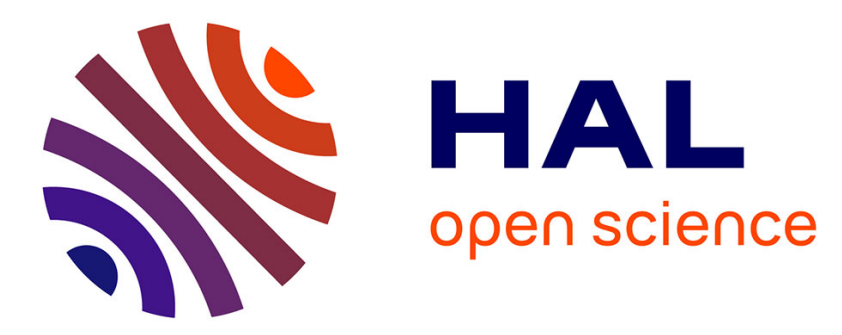

\title{
An affine formulation for the self-consistent modeling of elasto-viscoplastic heterogeneous materials based on the translated field method
}

Charles Moreau, Stéphane Berbenni

\section{- To cite this version:}

Charles Moreau, Stéphane Berbenni. An affine formulation for the self-consistent modeling of elastoviscoplastic heterogeneous materials based on the translated field method. International Journal of Plasticity, 2015, 64, pp.134-150. 10.1016/j.ijplas.2014.08.011 . hal-01513860

\section{HAL Id: hal-01513860 \\ https://hal.univ-lorraine.fr/hal-01513860}

Submitted on 30 Mar 2020

HAL is a multi-disciplinary open access archive for the deposit and dissemination of scientific research documents, whether they are published or not. The documents may come from teaching and research institutions in France or abroad, or from public or private research centers.
L'archive ouverte pluridisciplinaire HAL, est destinée au dépôt et à la diffusion de documents scientifiques de niveau recherche, publiés ou non, émanant des établissements d'enseignement et de recherche français ou étrangers, des laboratoires publics ou privés. 


\title{
An affine formulation for the self-consistent modeling of elasto-viscoplastic heterogeneous materials based on the translated field method
}

\author{
Charles Mareau ${ }^{a}$, Stéphane Berbenni ${ }^{\mathrm{b}}$ \\ a Arts et Métiers ParisTech, CER d'Angers, LAMPA, 2 bd du Ronceray, 49035 \\ Angers Cedex 1, France \\ ${ }^{\mathrm{b}}$ Laboratoire d'Etude des Microstructures et de Mécanique des Matériaux, LEM3, \\ UMR CNRS 7239, University of Lorraine, Ile du Saulcy, 57045 Metz, France
}

\begin{abstract}
The modeling of heterogeneous materials with an elasto-viscoplastic behavior is generally complex because of the differential nature of the local constitutive law. Indeed, the resolution of the heterogeneous problem involves space-time couplings which are generally difficult to estimate. In the present paper, a new homogenization model based on an affine linearization of the viscoplastic flow rule is proposed. First, the heterogeneous problem is written in the form of an integral equation. The purely thermoelastic and purely viscoplastic heterogeneous problems are solved independently using the self-consistent approximation. Using translated field techniques, the solutions of the above problems are combined to obtain the final self-consistent formulation. Then, some applications concerning two-phase fibre-reinforced composites and polycrystalline materials are presented. When compared to the reference solutions obtained from a FFT spectral method, a good description of the overall response of heterogeneous materials is obtained with the proposed model even when the viscoplastic flow rule is highly non-linear. Thanks to this approach, which is entirely formulated in the real-time space, the present model can be used for studying the response of heterogeneous materials submitted to complex thermo-mechanical loading paths with a good numerical efficiency.
\end{abstract}

Key words: A. Microstructures, B. Elastic-viscoplastic material, B. Fibre-reinforced composite, B. Polycrystalline material, C. Homogenization

Email address: charles.mareau@ensam.eu (Charles Mareau). 


\section{Introduction}

The self-consistent scheme is a common homogenization method that was developed to connect local deformation mechanisms to the overall behavior of heterogeneous disordered materials (Hershey, 1954; Kröner, 1958). In the past decades, many efforts have been made to obtain extensions of the selfconsistent approximation to the non-linear case. For heterogeneous materials with a rate-independent elastoplastic behavior, Kröner (1961) proposed a model where the plastic strain is considered as a stress-free strain (i.e. eigenstrain). However, internal stresses are largely overestimated with this method which, like Taylor's model (Taylor, 1938), leads to uniform plastic strains. As an alternative, Hill (1965) developed an incremental version of the selfconsistent approximation. The constitutive equations are written in the form of a pseudo-linear relation such that it is possible to use the self-consistent approximation at each stage of a loading path. Extending Hill's idea to the case of viscoplasticity, Hutchinson (1976) applied the self-consistent method by introducing a viscoplastic modulus which results from the linearization of the constitutive relation. This approach has been later modified by Molinari et al. (1987) and Lebensohn and Tomé (1993) who proposed an alternative definition of the viscoplastic modulus based upon a tangent, rather than secant, formulation. Later, a first order affine linearization procedure for non linear elastic, viscoplastic and elastoplastic composites and polycrystals was developed by Masson et al. (2000). In particular, these authors showed that such a first order affine approximation implemented within a self-consistent framework improves the estimate of the effective behavior of non-linear polycrystals in comparison with the incremental formulation of Hill (1965).

The case of elasto-viscoplasticity raises additional difficulties due to the differential nature of the constitutive equations involving different orders of time derivation. Different possibilities have been explored to overcome these difficulties. In the case of linear viscoelasticity, Hashin (1969) and Laws and McLaughlin (1978) have used the correspondence principle to propose a hereditary approach. Indeed, the use of Laplace-Carson transforms allows separating time and space variables such that it is possible to define a single viscoelastic modulus in the Laplace-Carson space. The self-consistent problem is solved in the Laplace-Carson space before proceeding to the inversion to the real time space. Extensions to the non-linear case have been proposed by Rougier et al. (1994) and Masson and Zaoui (1999). The constitutive law is first linearized at each step of the deformation path. In this way, the considered problem is converted into a viscoelastic problem, which can be classically solved using the self-consistent or the Mori-Tanaka approximation in the Laplace-Carson space. While the theoretical foundations of this approach are robust, the numerical implementation is rather complex mostly because the inversion of LaplaceCarson transforms requires intensive computations. Recently, the results of an 
enhanced affine formulation for two-phase composites were compared to numerical results obtained by the finite element method in Pierard and Doghri (2006) and Pierard et al. (2007).

In contrast with hereditary formulations, internal variable approaches can be preferred for the simplicity of their numerical implementation. Indeed, the resolution of the heterogeneous problem is achieved in the real time space with some internal variables whose introduction in the strain and stress concentration relations allows remembering the material's history. The first developments were carried out by Weng (1981a,b) who adapted Kröner's model to the case of elasto-viscoplasticity. However, similarly to the original proposition of Kröner (1961), internal stresses are largely overestimated with this method (Zaoui and Raphanel, 1993). This approach was later extended to the case of finite strains by Nemat-Nasser and Obata (1986) and Harren (1991). Mercier and Molinari (2009) used the additive interaction law developed by Molinari (Molinari et al., 1997; Molinari, 2002) to derive a self-consistent model based upon a tangent linearization of the viscoplastic flow rule. The additive interaction law has been later adapted to build a finite strain elasto-viscoplastic self-consistent model for polycrystals (Wang et al., 2010). Recently, an internal variable approach obtained from a variational method was developed by Lahellec and Suquet $(2007 a, b)$ to describe the behavior of composite materials. Paquin et al. (1999), Sabar et al. (2002) and Berbenni et al. (2004) proposed an alternative framework based upon the specific properties of projection operators. In the approach of Paquin et al. (1999), the purely elastic and purely viscoplastic heterogeneous problems are solved independently using the self-consistent approximation. Using translated field techniques, the individual solutions are combined to deduce a strain rate localization rule. Comparisons between both the translated field techniques and the additive interaction law were reported in Mercier et al. (2012) in the case of two-phase compressible or incompressible linear viscoelastic composites using a MoriTanaka approximation.

In the present paper, an extension of the translated field approach initiated by Paquin et al. (1999) for the modeling of disordered materials using the self-consistent approximation is proposed. The motivations are twofold. First, while the original approach of Paquin et al. (1999) derives from a classical secant approximation, this work aims at adopting an affine linearization procedure of the viscoplastic flow rule (i.e. a first order Taylor expansion of the viscoplastic flow rule) as suggested by Masson et al. (2000). Indeed, it has been observed that the first order affine formulation yields softer responses than the secant formulation (Masson and Zaoui, 1999; Masson et al., 2000; Molinari, 2002) which, in the case of pure viscoplasticity (or non linear elasticity in a general sense), may lead to the violation of a non-linear upper bound for the moduli (Gilormini, 1995). Second, in the original approach of Paquin et al. (1999), the local constitutive law only accounts for elastic and 
viscoplastic contributions. In the present work, the contribution of thermal expansion is also considered so that the proposed model may be used to investigate the response of disordered materials subjected to thermo-mechanical loading paths. Using the Mori-Tanaka approximation, a first order affine extension to the model of Paquin et al. (1999) has already been proposed for two-phase materials by Berbenni and Capolungo (2014). It was shown that predictions are very close to the ones obtained with the incremental variational approach developed by Lahellec and Suquet (2007b) for two-phase fibrereinforced composites. It is also thought that such translated field approaches are simpler to formulate than the recent incremental variational approaches for non-linear elastic-viscoplastic materials (Brassard et al., 2012; Lahellec and Suquet, 2007b, 2013) whose development within a self-consistent framework for polycrystalline materials has not been achieved yet.

The paper is structured as follows. In the first section, starting from field equations, the heterogeneous problem is written in the form of an integral equation. Then, the translated fields, which are obtained from the solutions of the purely thermoelastic and purely viscoplastic heterogeneous problems, are introduced in order to simplify the integral equation. Finally, the localization rule associated with the proposed affine formulation is deduced from the application of the self-consistent method. As an illustration, some applications are presented in the second section. The case of two-phase fibre-reinforced composites is first examined in order to compare the results from the proposed self-consistent approach with those given by others (Berbenni and Capolungo, 2014; Lahellec and Suquet, 2007b; Paquin et al., 1999). Then, the model is used to describe the behavior of polycrystalline materials when they are subjected to various thermomechanical loading conditions. To demonstrate the relevance of the proposed affine formulation, it is compared to the original classical secant formulation of Paquin et al. (1999) and to the full-field FFT spectral method of Moulinec and Suquet (1998).

\section{Model description}

\subsection{Field equations}

Considering a heterogeneous material, the proposed homogenization model aims at determining the macroscopic response of a representative volume element $V$ which is submitted to some specific boundary conditions. The macroscopic response is given by the relation between the macroscopic stress rate and strain rate tensors $(\dot{\Sigma}$ and $\dot{E})$. The macroscopic stress rate and strain rate tensors are connected to the local stress rate and strain rate tensors $(\dot{\sigma}$ and $\dot{\varepsilon})$ 
according to the classical averaging relations of homogenization theory:

$$
\begin{aligned}
& \dot{\Sigma}(t)=\frac{1}{V} \int_{V} \dot{\sigma}(x, t) d x=<\dot{\sigma}(t)> \\
& \dot{E}(t)=\frac{1}{V} \int_{V} \dot{\varepsilon}(x, t) d x=<\dot{\varepsilon}(t)>
\end{aligned}
$$

The determination of the local stress rate and strain rate fields is based on the resolution of the field equations associated with the heterogeneous problem. First, within the infinitesimal strain framework, the kinematical compatibility relation is given by:

$$
\dot{\varepsilon}(x, t)=\nabla_{s} \dot{u}(x, t)
$$

where $\dot{u}$ is the velocity field. In the absence of volume forces, static equilibrium conditions impose:

$$
\begin{aligned}
& \operatorname{div} \sigma(x, t)=0 \\
& \operatorname{div} \dot{\sigma}(x, t)=0
\end{aligned}
$$

Boundary conditions are prescribed on $\partial V$ according to:

$$
\dot{u}(x, t)=\dot{E}(t) . x \text { on } \partial V
$$

The decomposition of the total strain rate into themoelastic $\left(\dot{\varepsilon}_{t e}\right)$ and viscoplastic $\left(\dot{\varepsilon}_{v p}\right)$ contributions leads to:

$$
\dot{\varepsilon}(x, t)=\dot{\varepsilon}_{t e}(x, t)+\dot{\varepsilon}_{v p}(x, t)
$$

The thermoelastic strain rate results from a stress dependent contribution due to elasticity and a stress-independent contribution associated with thermal expansion $\dot{\varepsilon}_{t h}$ such that:

$$
\dot{\varepsilon}_{t e}(x, t)=s(x, t): \dot{\sigma}(x, t)+\dot{\varepsilon}_{t h}(x, t)
$$

where $\boldsymbol{s}$ (with $\boldsymbol{c}=\boldsymbol{s}^{-1}$ ) is the local elastic compliance tensor.

In the general case, the viscoplastic strain rate is a non-linear function $g$ depending on both the stress tensor $\sigma$ and some internal variables denoted by $\beta$. For further use of the self-consistent approximation, the viscoplastic flow rule has to be linearized. Different linearization methods have been proposed. In the original approach of Paquin et al. (1999), the flow rule linearization is based upon a secant formulation. However, since the secant formulation is known to overestimate the stiffness (Gilormini, 1995; Masson and Zaoui, 1999; Masson et al., 2000), the affine linearization method is chosen here. In its linearized form, the viscoplastic strain rate is written:

$$
\dot{\varepsilon}_{v p}(x, t)=g(\sigma, \beta) \approx \boldsymbol{m}_{t}(x, t): \sigma(x, t)+\dot{\eta}(x, t)
$$

where $\boldsymbol{m}_{t}$ (with $\boldsymbol{b}_{t}=\boldsymbol{m}_{t}^{-1}$ ) is the viscoplastic compliance tensor defined by:

$$
\boldsymbol{m}_{t}(x, t)=\frac{\partial g(\sigma, \beta)}{\partial \sigma}
$$


and $\dot{\eta}$ corresponds to a back-extrapolated strain rate:

$$
\dot{\eta}(x, t)=g(\sigma, \beta)-\boldsymbol{m}_{t}(x, t): \sigma(x, t)
$$

Thus, the final form of the constitutive law is:

$$
\dot{\varepsilon}(x, t)=\boldsymbol{s}(x, t): \dot{\sigma}(x, t)+\dot{\varepsilon}_{t h}(x, t)+\boldsymbol{m}_{t}(x, t): \sigma(x, t)+\dot{\eta}(x, t)
$$

The secant formulation proposed by Paquin et al. (1999) is retrieved when the tangent compliance tensor is replaced by the secant compliance tensor and by setting $\dot{\eta}$ to zero. Because of the differential nature of the above constitutive law, the modeling of elasto-viscoplastic heterogeneous solids requires to deal with a space-time relation which does not exist for elastic, elastoplastic or viscoplastic heterogeneous solids. The next section aims at proposing an integral equation, which includes the space-time coupling associated with the elasto-viscoplastic constitutive model, to describe the heterogeneous problem.

\subsection{Integral equation}

To recast the heterogeneous problem in the form of an integral equation, the local elastic modulus $\boldsymbol{c}$ (respectively viscoplastic modulus $\boldsymbol{b}_{t}$ ) is expressed as the sum of an uniform reference modulus $\boldsymbol{C}$ (respectively $\boldsymbol{B}_{t}$ ) and a fluctuating part $\delta \boldsymbol{c}$ (respectively $\delta \boldsymbol{b}_{t}$, the dependence with $t$ is omitted):

$$
\begin{gathered}
\boldsymbol{c}(x)=\boldsymbol{C}+\delta \boldsymbol{c}(x) \\
\boldsymbol{b}_{t}(x)=\boldsymbol{B}_{t}+\delta \boldsymbol{b}_{t}(x)
\end{gathered}
$$

To obtain the integral equation, the modified Green operators $\boldsymbol{\Gamma}^{C}$ and $\boldsymbol{\Gamma}^{B_{t}}$ associated with the thermoelastic and viscoplastic reference media are now introduced. The modified Green operators $\Gamma^{C}$ and $\Gamma^{B_{t}}$ are defined as follows:

$$
\begin{aligned}
& \Gamma_{i j k l}^{C}\left(x-x^{\prime}\right)=-\frac{1}{2}\left(G_{i k, j l}^{C}\left(x-x^{\prime}\right)+G_{j k, i l}^{C}\left(x-x^{\prime}\right)\right) \\
& \Gamma_{i j k l}^{B_{t}}\left(x-x^{\prime}\right)=-\frac{1}{2}\left(G_{i k, j l}^{B_{t}}\left(x-x^{\prime}\right)+G_{j k, i l}^{B_{t}}\left(x-x^{\prime}\right)\right)
\end{aligned}
$$

where $G^{C}$ (respectively $G^{B_{t}}$ ) is the elastic (respectively viscoplastic) Green function and, $x$ and $x^{\prime}$ are position vectors. The definition of Green functions can be found in many textbooks (Mura, 1987; Nemat-Nasser and Hori, 1994). As established by Kröner (1989), the modified Green operators display the following properties for any compatible strain (or strain rate) field and any balanced stress (or stress rate) field (in the following, the dependence with $x$ will be omitted):

$$
\begin{aligned}
& \Gamma^{C} * \dot{\sigma}=0 \\
& \Gamma^{B_{t}} * \sigma=0
\end{aligned}
$$




$$
\begin{gathered}
\left(\Gamma^{C}: C\right) * \dot{\varepsilon}=\dot{\varepsilon}-\dot{E} \\
\left(\Gamma^{B_{t}}: \boldsymbol{B}_{t}\right) * \dot{\varepsilon}=\dot{\varepsilon}-\dot{E}
\end{gathered}
$$

where $*$ denotes the spatial convolution product. Using the specific properties of the modified Green operators (equations (17) to (20)) in combination with the constitutive model (equation (12)), the integral equation associated with the heterogeneous problem is obtained:

$\dot{\varepsilon}=\dot{E}-\boldsymbol{\Gamma}^{C} *\left(\delta \boldsymbol{c}: \dot{\varepsilon}_{t e}-\boldsymbol{c}: \dot{\varepsilon}_{t h}\right)-\boldsymbol{\Gamma}^{B_{t}} *\left(\delta \boldsymbol{b}_{t}: \dot{\varepsilon}_{v p}-\boldsymbol{b}_{t}: \dot{\eta}\right)+\left(\boldsymbol{\Gamma}^{C}: \boldsymbol{C}-\boldsymbol{\Gamma}^{B_{t}}: \boldsymbol{B}_{t}\right) * \dot{\varepsilon}_{v p}$

The above integral equation, which contains the entire heterogeneous problem, is very similar to the one obtained by Paquin et al. (1999). The only difference lies in the choice of the linearization procedure. The integral equation shows how the local strain rate depends on both the macroscopic strain rate and the interactions between the different points of the representative volume element. In most cases, analytical solutions of this equation do not exist and some numerical procedures have to be used (e.g. spectral methods (Lebensohn et al., 2012; Moulinec and Suquet, 1998)). The self-consistent approximation, coupled with the translated field techniques, aims at estimating the unknown fields $\sigma, \dot{\sigma}$ and $\dot{\varepsilon}$ by considering only local interactions to simplify the integral equation. Following the approach of Paquin et al. (1999), the solutions of the purely elastic and purely viscoplastic heterogeneous problems will be used to introduce the translated fields in the integral equation.

\subsection{Cases of pure thermoelasticity and pure viscoplasticity}

Considering the same representative volume element with a purely thermoelastic behavior (i.e. $\dot{\varepsilon}=s: \dot{\sigma}+\dot{\varepsilon}_{t h}$ ) or a purely viscoplastic behavior (i.e. $\dot{\varepsilon}=\boldsymbol{m}_{t}: \sigma+\dot{\eta}$ ), the integral equation (21) simplifies to:

$$
\begin{gathered}
\dot{\varepsilon}=\dot{E}-\Gamma^{C} *\left(\delta \boldsymbol{c}: \dot{\varepsilon}-\boldsymbol{c}: \dot{\varepsilon}_{t h}\right) \text { for pure thermoelasticity } \\
\dot{\varepsilon}=\dot{E}-\Gamma^{B_{t}} *\left(\delta \boldsymbol{b}_{t}: \dot{\varepsilon}-\boldsymbol{b}_{t}: \dot{\eta}\right) \text { for pure viscoplasticity }
\end{gathered}
$$

Introducing the strain rate concentration tensors associated with the elastic and viscoplastic reference media $\left(\boldsymbol{A}^{C}\right.$ and $\left.\boldsymbol{A}^{B_{t}}\right)$, the above equations become:

$$
\begin{aligned}
& \dot{\varepsilon}=\boldsymbol{A}^{C}: \dot{E}+\boldsymbol{A}^{C}: \boldsymbol{\Gamma}^{C} *\left(\boldsymbol{c}: \dot{\varepsilon}_{t h}\right) \text { for pure thermoelasticity } \\
& \dot{\varepsilon}=\boldsymbol{A}^{B_{t}}: \dot{E}+\boldsymbol{A}^{B_{t}}: \boldsymbol{\Gamma}^{B_{t}} *\left(\boldsymbol{b}_{t}: \dot{\eta}\right) \text { for pure viscoplasticity }
\end{aligned}
$$

where $\boldsymbol{A}^{C}$ and $\boldsymbol{A}^{B_{t}}$ are defined as the solutions of the following equations:

$$
\begin{gathered}
\boldsymbol{A}^{C}+\Gamma^{C} *\left(\delta \boldsymbol{c}: \boldsymbol{A}^{C}\right)=\boldsymbol{I} \\
\boldsymbol{A}^{B_{t}}+\boldsymbol{\Gamma}^{B_{t}} *\left(\delta \boldsymbol{b}_{t}: \boldsymbol{A}^{B_{t}}\right)=\boldsymbol{I}
\end{gathered}
$$


The macroscopic constitutive laws display the same structure as the local constitutive laws of the heterogeneous media such that:

$$
\begin{gathered}
\dot{\Sigma}=C^{e}:\left(\dot{E}-\dot{E}_{t h}^{e}\right) \text { for pure thermoelasticity } \\
\Sigma=\boldsymbol{B}_{t}^{e}:\left(\dot{E}-\dot{N}^{e}\right) \text { for pure viscoplasticity }
\end{gathered}
$$

where $\boldsymbol{C}^{e}, \boldsymbol{B}_{t}^{e}, \dot{E}_{t h}^{e}$ and $\dot{N}^{e}$ are respectively the effective elastic modulus, the effective viscoplastic modulus, the effective thermal strain rate and the effective back-extrapolated strain rate of the heterogeneous solid with either a purely thermoelastic behavior or a purely viscoplastic behavior. The stress (rate) concentration rules are deduced from relations (24) and (25):

$$
\begin{aligned}
\dot{\sigma} & =\boldsymbol{c}: \boldsymbol{A}^{C}: \boldsymbol{S}^{e}: \dot{\Sigma}+\boldsymbol{c}: \boldsymbol{A}^{C}: \dot{E}_{t h}^{e} \\
& +\boldsymbol{c}: \boldsymbol{A}^{C}: \boldsymbol{\Gamma}^{C} *\left(\boldsymbol{c}: \dot{\varepsilon}_{t h}\right)-\boldsymbol{c}: \dot{\varepsilon}_{t h} \text { for pure thermoelasticity } \\
\sigma & =\boldsymbol{b}_{t}: \boldsymbol{A}^{B_{t}}: \boldsymbol{M}_{t}^{e}: \Sigma+\boldsymbol{b}_{t}: \boldsymbol{A}^{B_{t}}: \dot{N}^{e} \\
& +\boldsymbol{b}_{t}: \boldsymbol{A}^{B_{t}}: \boldsymbol{\Gamma}^{B_{t}} *\left(\boldsymbol{b}_{t}: \dot{\eta}\right)-\boldsymbol{b}_{t}: \dot{\eta} \text { for pure viscoplasticity }
\end{aligned}
$$

with $\boldsymbol{S}^{e}=\boldsymbol{C}^{e-1}$ and $\boldsymbol{M}_{t}^{e}=\boldsymbol{B}_{t}^{e-1}$. The averaging conditions $<\dot{\varepsilon}>=\dot{E}$, $<\dot{\sigma}\rangle=\dot{\Sigma}$ and $\langle\sigma\rangle=\Sigma$ impose:

$$
\begin{gathered}
\boldsymbol{C}^{e}=<\boldsymbol{c}: \boldsymbol{A}^{C}> \\
\boldsymbol{B}_{t}^{e}=<\boldsymbol{b}_{t}: \boldsymbol{A}^{B_{t}}> \\
<\boldsymbol{A}^{C}>=\boldsymbol{I} \\
<\boldsymbol{A}^{B_{t}}>=\boldsymbol{I} \\
<\boldsymbol{A}^{C}: \boldsymbol{\Gamma}^{C} *\left(\boldsymbol{c}: \dot{\varepsilon}_{t h}\right)>=0 \\
<\boldsymbol{A}^{B_{t}}: \boldsymbol{\Gamma}^{B_{t}} *\left(\boldsymbol{b}_{t}: \dot{\eta}\right)>=0 \\
<\boldsymbol{c}: \dot{\varepsilon}_{t h}>-\boldsymbol{C}^{e}: \dot{E}_{t h}^{e}=<\boldsymbol{c}: \boldsymbol{A}^{C}: \boldsymbol{\Gamma}^{C} *\left(\boldsymbol{c}: \dot{\varepsilon}_{t h}\right)> \\
<\boldsymbol{b}_{t}: \dot{\eta}>-\boldsymbol{B}_{t}^{e}: \dot{N}^{e}=<\boldsymbol{b}_{t}: \boldsymbol{A}^{B_{t}}: \boldsymbol{\Gamma}^{B_{t}} *\left(\boldsymbol{b}_{t}: \dot{\eta}\right)>
\end{gathered}
$$

\subsection{Introduction of the translated fields}

The integral equation (21) can be simplified by using the solutions of the purely thermoelastic and purely viscoplastic heterogeneous problems. Considering the thermoelastic part of the strain rate, it can be decomposed into the solution of the purely thermoelastic problem (denoted by $\dot{e}^{\prime}$ ) and a residual strain rate field corresponding to the internal stress rate field $\dot{\sigma}^{\prime}$ :

$$
\dot{\varepsilon}_{t e}=s: \dot{\sigma}+\dot{\varepsilon}_{t h}=\dot{e}^{\prime}+s: \dot{\sigma}^{\prime}
$$


where $\dot{e}^{\prime}$ is taken as the solution of the purely thermoelastic heterogeneous problem:

$$
\dot{e}^{\prime}=<\dot{\varepsilon}_{t e}>-\Gamma^{C} *\left(\delta \boldsymbol{c}: \dot{e}^{\prime}-\boldsymbol{c}: \dot{\varepsilon}_{t h}\right)
$$

Adopting the same reasoning for the viscoplastic strain rate yields:

$$
\dot{\varepsilon}_{v p}=\boldsymbol{m}_{t}: \sigma+\dot{\eta}=\dot{e}^{\prime \prime}+\boldsymbol{m}_{t}: \sigma^{\prime \prime}
$$

where $\dot{e}^{\prime \prime}$ is chosen as the solution of the purely viscoplastic heterogeneous problem:

$$
\dot{e}^{\prime \prime}=<\dot{\varepsilon}_{v p}>-\boldsymbol{\Gamma}^{B_{t}} *\left(\delta \boldsymbol{b}_{t}: \dot{e}^{\prime \prime}-\boldsymbol{b}_{t}: \dot{\eta}\right)
$$

Because of the additive decomposition of the strain rate into thermoelastic and viscoplastic parts, one obtains:

$$
\dot{\varepsilon}=\dot{\varepsilon}_{t e}+\dot{\varepsilon}_{v p}=\dot{e}^{\prime}+\dot{e}^{\prime \prime}+\dot{\varepsilon}^{\star}
$$

where $\dot{\varepsilon}^{\star}$ is the translated strain rate field associated to the translated stress (rate) fields $\dot{\sigma}^{\prime}$ and $\sigma^{\prime \prime}$ :

$$
\dot{\varepsilon}^{\star}=s: \dot{\sigma}^{\prime}+\boldsymbol{m}_{t}: \sigma^{\prime \prime}
$$

The most interesting feature of this decomposition lies in the specific properties of the recently introduced translated fields. Indeed, $\dot{\sigma}^{\prime}$ and $\sigma^{\prime \prime}$ are balanced stress (rate) fields while $\dot{\varepsilon}^{\star}$ is compatible. Also, the importance of the translated strain rate field $\dot{\varepsilon}^{\star}$ should be highlighted. According to equation (44), the total strain rate is decomposed into the thermoelastic solution $\left(\dot{e}^{\prime}\right)$, the viscoplastic solution $\left(\dot{e}^{\prime \prime}\right)$ and an additional contribution $\left(\dot{\varepsilon}^{\star}\right)$ which represents the complexity of the space-time interactions associated with the thermoelastic and viscoplastic couplings. This treatment of the entire problem allows for combining the separate solutions of the thermoelastic and viscoplastic heterogeneous problem without compromising the issue of their mutual interactions which are described through $\dot{\varepsilon}^{\star}$.

Based on the translated field properties, an associated problem, which is described by the following integral equation, is now considered:

$\dot{\varepsilon}^{\star}=\dot{E}^{\star}-\boldsymbol{\Gamma}^{C} *\left(\delta \boldsymbol{c}: \boldsymbol{s}: \dot{\sigma}^{\prime}\right)-\boldsymbol{\Gamma}^{B_{t}} *\left(\delta \boldsymbol{b}_{t}: \boldsymbol{m}_{t}: \sigma^{\prime \prime}\right)+\left(\boldsymbol{\Gamma}^{C}: \boldsymbol{C}-\boldsymbol{\Gamma}^{B_{t}}: \boldsymbol{B}_{t}\right) *\left(\boldsymbol{m}_{t}: \sigma^{\prime \prime}\right)$

where $\dot{E}^{\star}$ is the macroscopic strain rate field from which boundary conditions are prescribed on $\partial V$. Also, because of the properties given by equations (32) to (39), the following averaging relations are verified:

$$
\begin{gathered}
<\dot{\varepsilon}^{\star}>=\dot{E}^{\star}=0 \\
<s: \dot{\sigma}^{\prime}>=0 \\
<\boldsymbol{m}_{t}: \sigma^{\prime \prime}>=0 \\
\boldsymbol{S}^{e}:<\dot{\sigma}^{\prime}>=\left(\boldsymbol{S}^{e}: \dot{\Sigma}+\dot{E}_{t h}^{e}\right)-<\dot{\varepsilon}_{t e}>=<\dot{\varepsilon}_{v p}>-\dot{E}_{v p}^{e} \\
\boldsymbol{M}_{t}^{e}:<\sigma^{\prime \prime}>=\left(\boldsymbol{M}_{t}^{e}: \Sigma+\dot{N}^{e}\right)-<\dot{\varepsilon}_{v p}>
\end{gathered}
$$




\subsection{Self-consistent approximation}

In order to solve the associated problem, which consists of equations (41), (43) and (46), the self-consistent approximation is used. The main idea of the self-consistent approximation is the decomposition of the modified Green operator $\Gamma^{C}$ (respectively $\boldsymbol{\Gamma}^{B_{t}}$ ) associated with the thermoelastic (respectively viscoplastic) reference homogeneous medium into a local part (subscript $l$ ) and a non-local part (subscript $n l$ ) to weaken the influence of non-local interactions:

$$
\begin{aligned}
\Gamma^{C}\left(x-x^{\prime}\right) & =\Gamma_{l}^{C} \delta\left(x-x^{\prime}\right)+\Gamma_{n l}^{C}\left(x-x^{\prime}\right) \\
\Gamma^{B_{t}}\left(x-x^{\prime}\right) & =\boldsymbol{\Gamma}_{l}^{B_{t}} \delta\left(x-x^{\prime}\right)+\boldsymbol{\Gamma}_{n l}^{B_{t}}\left(x-x^{\prime}\right)
\end{aligned}
$$

The influence of non-local terms, which are difficult to estimate, is weakened by introducing uniform second-order tensors in the integral equations (41), (43) and (46). Indeed, for any uniform tensor $X$, the fields $\boldsymbol{A}^{B_{t}}: X, \boldsymbol{A}^{C}: X$ and $X$ are kinematically admissible while the fields $c: \boldsymbol{A}^{C}: X$ and $\boldsymbol{b}_{t}: \boldsymbol{A}^{B_{t}}: X$ are balanced. Thus, these different fields benefit from the specific properties of the modified Green operators $\boldsymbol{\Gamma}^{C}$ and $\boldsymbol{\Gamma}^{B_{t}}$ (relations (17) to (20)). Therefore, using the above decomposition of the modified Green operators and introducing the unknown uniform tensors $\left(Y_{1}\right.$ to $\left.Y_{5}\right)$ in the integral equations (41), (43) and (46) leads to:

$$
\begin{aligned}
\dot{\varepsilon}^{\star} & =\dot{E}^{\star}-\boldsymbol{\Gamma}_{l}^{C}:\left(\delta \boldsymbol{c}: \boldsymbol{s}: \dot{\sigma}^{\prime}-\boldsymbol{c}: \boldsymbol{A}^{C}: Y_{1}\right) \\
& -\boldsymbol{\Gamma}_{n l}^{C} *\left(\delta \boldsymbol{c}: \boldsymbol{s}: \dot{\sigma}^{\prime}-\boldsymbol{c}: \boldsymbol{A}^{C}: Y_{1}\right) \\
& -\boldsymbol{\Gamma}_{l}^{B_{t}}:\left(\delta \boldsymbol{b}_{t}: \boldsymbol{m}_{t}: \sigma^{\prime \prime}-\boldsymbol{b}_{t}: \boldsymbol{A}^{B_{t}}: Y_{2}\right) \\
& -\boldsymbol{\Gamma}_{n l}^{B_{t}} *\left(\delta \boldsymbol{b}_{t}: \boldsymbol{m}_{t}: \sigma^{\prime \prime}-\boldsymbol{b}_{t}: \boldsymbol{A}^{B_{t}}: Y_{2}\right) \\
& +\left(\boldsymbol{\Gamma}_{l}^{C}: \boldsymbol{C}-\boldsymbol{\Gamma}_{l}^{B_{t}}: \boldsymbol{B}_{t}\right):\left(\boldsymbol{m}_{t}: \sigma^{\prime \prime}-\boldsymbol{A}^{B_{t}}: Y_{3}\right) \\
& +\left(\boldsymbol{\Gamma}_{n l}^{C}: \boldsymbol{C}-\boldsymbol{\Gamma}_{n l}^{B_{t}}: \boldsymbol{B}_{t}\right) *\left(\boldsymbol{m}_{t}: \sigma^{\prime \prime}-\boldsymbol{A}^{B_{t}}: Y_{3}\right) \\
& \dot{e}^{\prime}=<\dot{\varepsilon}_{t e}>-\boldsymbol{\Gamma}_{l}^{C}:\left(\delta \boldsymbol{c}: \dot{e}^{\prime}-\boldsymbol{c}: \dot{\varepsilon}_{t h}+\boldsymbol{C}: Y_{4}\right) \\
& -\boldsymbol{\Gamma}_{n l}^{C} *\left(\delta \boldsymbol{c}: \dot{e}^{\prime}-\boldsymbol{c}: \dot{\varepsilon}_{t h}+\boldsymbol{C}: Y_{4}\right) \\
\dot{e}^{\prime \prime} & =<\dot{\varepsilon}_{v p}>-\boldsymbol{\Gamma}_{l}^{B_{t}}:\left(\delta \boldsymbol{b}_{t}: \dot{e}^{\prime \prime}-\boldsymbol{b}_{t}: \dot{\eta}+\boldsymbol{B}_{t}: Y_{5}\right) \\
& -\boldsymbol{\Gamma}_{n l}^{B_{t}} *\left(\delta \boldsymbol{b}_{t}: \dot{e}^{\prime \prime}-\boldsymbol{b}_{t}: \dot{\eta}+\boldsymbol{B}_{t}: Y_{5}\right)
\end{aligned}
$$

Neglecting the non-local terms requires to choose $Y_{1}, Y_{2}, Y_{3}, Y_{4}$ and $Y_{5}$ such that the following self-consistency conditions are fulfilled:

$$
\begin{gathered}
<\delta \boldsymbol{c}: \boldsymbol{s}: \dot{\sigma}^{\prime}-\boldsymbol{c}: \boldsymbol{A}^{C}: Y_{1}>=0 \text { so } Y_{1}=\boldsymbol{S}^{e}:<\dot{\sigma}^{\prime}> \\
<\delta \boldsymbol{b}_{t}: \boldsymbol{m}_{t}: \sigma^{\prime \prime}-\boldsymbol{b}_{t}: \boldsymbol{A}^{B_{t}}: Y_{2}>=0 \text { so } Y_{2}=\boldsymbol{M}_{t}^{e}:<\sigma^{\prime \prime}> \\
\quad<\boldsymbol{m}_{t}: \sigma^{\prime \prime}-\boldsymbol{A}^{B_{t}}: Y_{3}>=0 \text { so } Y_{3}=0 \\
<\delta \boldsymbol{c}: \dot{e}^{\prime}-\boldsymbol{c}: \dot{\varepsilon}_{t h}+\boldsymbol{C}: Y_{4}>=0 \text { so } \boldsymbol{C}: Y_{4}=\boldsymbol{C}^{e}: \dot{E}_{t h}^{e}
\end{gathered}
$$




$$
<\delta \boldsymbol{b}_{t}: \dot{e}^{\prime \prime}-\boldsymbol{b}_{t}: \dot{\eta}+\boldsymbol{B}_{t}: Y_{5}>=0 \text { so } \boldsymbol{B}_{t}: Y_{5}=\boldsymbol{B}_{t}^{e}: \dot{N}^{e}
$$

Considering only local terms and using the expressions of the unknown tensors, the self-consistent solution of the associated problem is given by:

$$
\begin{aligned}
& \dot{\varepsilon}^{\star}=\dot{E}^{\star}-\boldsymbol{\Gamma}_{l}^{C}:\left(\delta \boldsymbol{c}: \boldsymbol{s}: \dot{\sigma}^{\prime}-\boldsymbol{c}: \boldsymbol{A}^{C}: \boldsymbol{S}^{e}:<\dot{\sigma}^{\prime}>\right) \\
&-\boldsymbol{\Gamma}_{l}^{B_{t}}:\left(\delta \boldsymbol{b}_{t}: \boldsymbol{m}_{t}: \sigma^{\prime \prime}-\boldsymbol{b}_{t}: \boldsymbol{A}^{B_{t}}: \boldsymbol{M}_{t}^{e}:<\sigma^{\prime \prime}>\right) \\
&+\left(\boldsymbol{\Gamma}_{l}^{C}: \boldsymbol{C}-\boldsymbol{\Gamma}_{l}^{B_{t}}: \boldsymbol{B}_{t}\right):\left(\boldsymbol{m}_{t}: \sigma^{\prime \prime}\right) \\
& \dot{e}^{\prime}=<\dot{\varepsilon}_{t e}>-\boldsymbol{\Gamma}_{l}^{C}:\left(\delta \boldsymbol{c}: \dot{e}^{\prime}-\boldsymbol{c}: \dot{\varepsilon}_{t h}+\boldsymbol{C}^{e}: \dot{E}_{t h}^{e}\right) \\
& \dot{e}^{\prime \prime}=<\dot{\varepsilon}_{v p}>-\boldsymbol{\Gamma}_{l}^{B_{t}}:\left(\delta \boldsymbol{b}_{t}: \dot{e}^{\prime \prime}-\boldsymbol{b}_{t}: \dot{\eta}+\boldsymbol{B}_{t}^{e}: \dot{N}^{e}\right)
\end{aligned}
$$

Regarding the properties of the elastic and viscoplastic reference media, it is imposed to choose the solutions obtained for the purely thermoelastic and purely viscoplastic heterogeneous problems using the self-consistent approximation (i.e. $\boldsymbol{C}=\boldsymbol{C}^{e}$ and $\boldsymbol{B}_{t}=\boldsymbol{B}_{t}^{e}$ ). This choice for the properties of the reference media is needed to ensure that the averaging relations $\left\langle\dot{e}^{\prime}\right\rangle=\left\langle\dot{\varepsilon}_{t e}\right\rangle$ and $<\dot{e}^{\prime \prime}>=<\varepsilon_{v p}>$ are verified.

Returning to the original problem, the strain rate concentration rule is obtained from the combination of equations (44), (62), (63) and (64):

$$
\begin{aligned}
\dot{\varepsilon} & =\boldsymbol{A}^{C}: \dot{E}+\boldsymbol{A}^{C}: \boldsymbol{\Gamma}_{l}^{C}:\left(\boldsymbol{c}: \dot{\varepsilon}_{t h}-\boldsymbol{C}^{e}: \dot{E}_{t h}^{e}\right)+\boldsymbol{A}^{C}: \boldsymbol{\Gamma}_{l}^{B_{t}}:\left(\boldsymbol{b}_{t}: \dot{\eta}-\boldsymbol{B}_{t}^{e}: \dot{N}^{e}\right) \\
& +\boldsymbol{A}^{C}: \boldsymbol{\Gamma}_{l}^{C}:\left(\delta \boldsymbol{c}: \dot{\varepsilon}^{v p}+\boldsymbol{c}: \boldsymbol{A}^{C}:\left(<\dot{\varepsilon}_{v p}>-\dot{E}_{v p}^{e}\right)\right) \\
& -\boldsymbol{A}^{C}: \boldsymbol{\Gamma}_{l}^{B_{t}}:\left(\delta \boldsymbol{b}_{t}: \dot{\varepsilon}^{v p}-\boldsymbol{b}_{t}: \boldsymbol{A}^{B_{t}}:\left(\boldsymbol{M}_{t}^{e}: \Sigma+\dot{N}^{e}-<\dot{\varepsilon}_{v p}>\right)\right) \\
& +\boldsymbol{A}^{C}:\left(\boldsymbol{\Gamma}_{l}^{C}: \boldsymbol{C}^{e}-\boldsymbol{\Gamma}_{l}^{B_{t}}: \boldsymbol{B}_{t}^{e}\right):\left(\dot{\varepsilon}_{v p}-\boldsymbol{A}^{B_{t}}:<\dot{\varepsilon}_{v p}>\right) \\
& -\boldsymbol{A}^{C}:\left(\boldsymbol{\Gamma}_{l}^{C}: \boldsymbol{C}^{e}-\boldsymbol{\Gamma}_{l}^{B_{t}}: \boldsymbol{B}_{t}^{e}\right):\left(\boldsymbol{A}^{B_{t}}: \boldsymbol{\Gamma}_{l}^{B_{t}}:\left(\boldsymbol{b}_{t}: \dot{\eta}-\boldsymbol{B}_{t}^{e}: \dot{N}^{e}\right)\right)
\end{aligned}
$$

The above relation describes the connection between $\dot{\varepsilon}$ and $\dot{E}$ as a function of the spatial interactions resulting from the thermal and viscoplastic incompatibilities. It is emphasized that only the self-consistent solutions of the purely thermoelastic and viscoplastic heterogeneous problems are needed to estimate the local strain rate and stress rate fields. Moreover, in contrast with the propositions of Rougier et al. (1994) and Masson and Zaoui (1999), no complex numerical procedure is involved since relations (63) to (65) are entirely formulated in the real-time space. Substituting the concentration rule (65) in the local constitutive equation (12) gives the following interaction law for 
stresses:

$$
\begin{aligned}
\dot{\sigma} & =\boldsymbol{c}: \boldsymbol{A}^{C}:\left(\boldsymbol{S}^{e}: \dot{\Sigma}+\dot{E}_{v p}^{e}+\dot{E}_{t h}^{e}\right)-\boldsymbol{c}: \dot{\varepsilon}^{v p}-\boldsymbol{c}: \dot{\varepsilon}^{t h} \\
& +\boldsymbol{c}: \boldsymbol{A}^{C}: \boldsymbol{\Gamma}_{l}^{C}:\left(\boldsymbol{c}: \dot{\varepsilon}_{t h}-\boldsymbol{C}^{e}: \dot{E}_{t h}^{e}\right)+\boldsymbol{c}: \boldsymbol{A}^{C}: \boldsymbol{\Gamma}_{l}^{B_{t}}:\left(\boldsymbol{b}_{t}: \dot{\eta}-\boldsymbol{B}_{t}^{e}: \dot{N}^{e}\right) \\
& +\boldsymbol{c}: \boldsymbol{A}^{C}: \boldsymbol{\Gamma}_{l}^{C}:\left(\delta \boldsymbol{c}: \dot{\varepsilon}^{v p}+\boldsymbol{c}: \boldsymbol{A}^{C}:\left(<\dot{\varepsilon}_{v p}>-\dot{E}_{v p}^{e}\right)\right) \\
& -\boldsymbol{c}: \boldsymbol{A}^{C}: \boldsymbol{\Gamma}_{l}^{B_{t}}:\left(\delta \boldsymbol{b}_{t}: \dot{\varepsilon}^{v p}-\boldsymbol{b}_{t}: \boldsymbol{A}^{B_{t}}:\left(\boldsymbol{M}_{t}^{e}: \Sigma+\dot{N}^{e}-<\dot{\varepsilon}_{v p}>\right)\right) \\
& +\boldsymbol{c}: \boldsymbol{A}^{C}:\left(\boldsymbol{\Gamma}_{l}^{C}: \boldsymbol{C}^{e}-\boldsymbol{\Gamma}_{l}^{B_{t}}: \boldsymbol{B}_{t}^{e}\right):\left(\dot{\varepsilon}_{v p}-\boldsymbol{A}^{B_{t}}:<\dot{\varepsilon}_{v p}>\right) \\
& -\boldsymbol{c}: \boldsymbol{A}^{C}:\left(\boldsymbol{\Gamma}_{l}^{C}: \boldsymbol{C}^{e}-\boldsymbol{\Gamma}_{l}^{B_{t}}: \boldsymbol{B}_{t}^{e}\right):\left(\boldsymbol{A}^{B_{t}}: \boldsymbol{\Gamma}_{l}^{B_{t}}:\left(\boldsymbol{b}_{t}: \dot{\eta}-\boldsymbol{B}_{t}^{e}: \dot{N}^{e}\right)\right)
\end{aligned}
$$

where $\dot{E}_{v p}^{e}$ is the effective viscoplastic strain rate which is chosen to impose the condition $\langle\dot{\sigma}\rangle=\dot{\Sigma}$ :

$$
\begin{aligned}
\dot{E}_{v p}^{e} & =\boldsymbol{Z}:<\boldsymbol{c}: \dot{\varepsilon}^{v p}>-\boldsymbol{Z}:<\boldsymbol{c}: \boldsymbol{A}^{C}: \boldsymbol{\Gamma}_{l}^{B_{t}}:\left(\boldsymbol{b}_{t}: \dot{\eta}-\boldsymbol{B}_{t}^{e}: \dot{N}^{e}\right)> \\
& -\boldsymbol{Z}:<\boldsymbol{c}: \boldsymbol{A}^{C}: \boldsymbol{\Gamma}_{l}^{C}:\left(\delta \boldsymbol{c}: \dot{\varepsilon}^{v p}+\boldsymbol{c}: \boldsymbol{A}^{C}:<\dot{\varepsilon}_{v p}>\right)> \\
& +\boldsymbol{Z}:<\boldsymbol{c}: \boldsymbol{A}^{C}: \boldsymbol{\Gamma}_{l}^{B_{t}}:\left(\delta \boldsymbol{b}_{t}: \dot{\varepsilon}^{v p}-\boldsymbol{b}_{t}: \boldsymbol{A}^{B_{t}}:\left(\boldsymbol{M}_{t}^{e}: \Sigma+\dot{N}^{e}-<\dot{\varepsilon}_{v p}>\right)\right)> \\
& -\boldsymbol{Z}:<\boldsymbol{c}: \boldsymbol{A}^{C}:\left(\boldsymbol{\Gamma}_{l}^{C}: \boldsymbol{C}^{e}-\boldsymbol{\Gamma}_{l}^{B_{t}}: \boldsymbol{B}_{t}^{e}\right):\left(\dot{\varepsilon}_{v p}-\boldsymbol{A}^{B_{t}}:<\dot{\varepsilon}_{v p}>\right)> \\
& +\boldsymbol{Z}:<\boldsymbol{c}: \boldsymbol{A}^{C}:\left(\boldsymbol{\Gamma}_{l}^{C}: \boldsymbol{C}^{e}-\boldsymbol{\Gamma}_{l}^{B_{t}}: \boldsymbol{B}_{t}^{e}\right):\left(\boldsymbol{A}^{B_{t}}: \boldsymbol{\Gamma}_{l}^{B_{t}}:\left(\boldsymbol{b}_{t}: \dot{\eta}-\boldsymbol{B}_{t}^{e}: \dot{N}^{e}\right)\right)>
\end{aligned}
$$

with:

$$
Z=\left(I-\boldsymbol{S}^{e}:<\boldsymbol{c}: \boldsymbol{A}^{C}: \boldsymbol{\Gamma}_{l}^{C}: \boldsymbol{c}: \boldsymbol{A}^{C}>\right)^{-1}: \boldsymbol{S}^{e}
$$

\subsection{Particular case of two-phase materials}

In the case of two-phase materials (with phases $r=1,2$ ), the Levin's formula (Levin, 1967) can be used to further simplify the strain rate concentration equation (65) (see also Masson et al. (2000); Rekik et al. (2007)). Thus, for each phase $r=1,2$, the expression of $\dot{e}^{\prime}(r)$ simplifies to:

$$
\dot{e}^{\prime(r)}=A^{C^{(r)}}:<\dot{\varepsilon}_{t e}>+a^{C(r)}
$$

where $a^{C^{(r)}}$ is given by:

$$
a^{C^{(r)}}=\left[\boldsymbol{A}^{\boldsymbol{C}^{(r)}}-\boldsymbol{I}\right]:\left(\boldsymbol{c}^{(2)}-\boldsymbol{c}^{(1)}\right)^{-1}:\left(\tau_{t e}^{(2)}-\tau_{t e}^{(1)}\right)
$$

with $\tau_{t e}{ }^{(r)}=-\boldsymbol{b}_{\boldsymbol{t}}{ }^{(r)}: \dot{\varepsilon}_{t h}^{(r)}$. In a similar manner, the expression of $\dot{e}^{\prime \prime}(r)$ becomes:

$$
\dot{e}^{\prime \prime(r)}=\boldsymbol{A}^{B_{t}^{(r)}}:<\dot{\varepsilon}_{v p}>+a^{B_{t}(r)}
$$


where $a^{B_{t}(r)}$ is given by:

$$
a^{B_{t}(r)}=\left[\boldsymbol{A}^{\boldsymbol{B}_{\boldsymbol{t}}(r)}-\boldsymbol{I}\right]:\left(\boldsymbol{b}_{\boldsymbol{t}}^{(2)}-\boldsymbol{b}_{\boldsymbol{t}}^{(1)}\right)^{-1}:\left(\tau_{v p}{ }^{(2)}-\tau_{v p}{ }^{(1)}\right)
$$

with $\tau_{v p}{ }^{(r)}=-\boldsymbol{b}_{\boldsymbol{t}}{ }^{(r)}: \dot{\eta}^{(r)}$. Thus, for two-phase materials, equation (65) can be recast for each phase $r=1,2$ as (the superscript $(r)$ is now removed to simplify the notations):

$$
\begin{aligned}
\dot{\varepsilon} & =\boldsymbol{A}^{C}: \dot{E}+a^{C}+\boldsymbol{A}^{C}:\left(\boldsymbol{A}^{B_{t}}\right)^{-1}: a^{B_{t}} \\
& +\boldsymbol{A}^{C}: \boldsymbol{\Gamma}_{l}^{C}:\left(\delta \boldsymbol{c}: \dot{\varepsilon}^{v p}+\boldsymbol{c}: \boldsymbol{A}^{C}:\left(<\dot{\varepsilon}_{v p}>-\dot{E}_{v p}^{e}\right)\right) \\
& -\boldsymbol{A}^{C}: \boldsymbol{\Gamma}_{l}^{B_{t}}:\left(\delta \boldsymbol{b}_{t}: \dot{\varepsilon}^{v p}+\boldsymbol{b}_{t}: \boldsymbol{A}^{B_{t}}:<\left(\delta \boldsymbol{m}_{t}:\left(\sigma-\tau_{v p}-\boldsymbol{b}_{t}: a^{B_{t}}\right)\right)>\right) \\
& +\boldsymbol{A}^{C}:\left(\boldsymbol{\Gamma}_{l}^{C}: \boldsymbol{C}^{e}-\boldsymbol{\Gamma}_{l}^{B_{t}}: \boldsymbol{B}_{t}^{e}\right):\left(\dot{\varepsilon}_{v p}-\boldsymbol{A}^{B_{t}}:<\dot{\varepsilon}_{v p}>-a^{B_{t}}\right)
\end{aligned}
$$

where $\dot{E}_{v p}^{e}$ is obtained from equation (67) together with equation (73) and averaging relations (1) and (2).

\section{Applications}

\subsection{Fibre-reinforced composites}

In order to study the effect of non-linear viscosity on the overall response, the present self-consistent (SC) approximation, which uses the translated field (TF) techniques together with a first order affine (AFF) linearization procedure of the viscoplastic flow rule (equation (73), labeled as SC-TF-AFF), is applied to two-phase fibre-reinforced composites. The interest for studying such composite materials is twofold. First, a Mori-Tanaka approximation based on translated field techniques and coupled with a first order affine formulation (labeled as MT-TF-AFF) has been recently developed by Berbenni and Capolungo (2014). It is therefore interesting to compare the self-consistent and Mori-Tanaka schemes for two-phase composite materials with an elastoviscoplastic behavior. Second, to observe the influence of the linearization procedure, the present SC-TF-AFF approach is also compared to the original self-consistent approach of Paquin et al. (1999) (labeled as SC-TF-SEC) which uses a secant approximation of the viscoplastic flow rule (Paquin et al., 1999).

The composite material is made of a matrix phase $(r=M)$ and long cylindrical fibres $(r=I)$. Fibres are aligned along the same direction $\left(x_{3}\right)$ and are perpendicular to the $\left(x_{1}, x_{2}\right)$ plane. These microstructures were extensively studied by Lahellec and Suquet (2007b) with a variational approach (labeled 
as SEC (EIV+HS)) developed for non-linear composites. This variational approach consists of an incremental effective potential approach where the internal variables are the effective viscoplastic strain rates (i.e. EIV stands for "Effective Internal Variable") which are determined from a modified secant approach. The effective properties of the thermoelastic-like homogeneous equivalent medium are obtained from the Hashin-Shtrikman (HS) estimates calculated for the composite cylinder assemblage (CCA). For such fibre-reinforced composites, full-field FFT calculations have been performed earlier by Lahellec and Suquet (2007b) to obtain "exact" solutions. Their results are used here as reference solutions to demonstrate the relevance of the present affine approach. The RVE studied by Lahellec and Suquet (2007b) for the FFT calculations is a $2 \mathrm{D}$ volume element where fibres are seen as a composite cylinder assemblage of self-similar cylinders.

Here, both phases are given an elasto-viscoplastic behavior (with $\dot{\varepsilon}_{t h}=0$ ). The non-linear viscoplastic flow rule takes the form of a power law (Norton's model):

$$
\dot{\varepsilon}=s: \dot{\sigma}+\frac{3}{2} \dot{\varepsilon_{0}}\left(\frac{\sigma_{e q}}{\sigma_{0}^{(r)}}\right)^{1 / m} \frac{s}{\sigma_{e q}}
$$

where $m$ is the strain-rate sensitivity parameter which is the same for both phases, $s$ is the deviatoric stress, $\sigma_{e q}$ is the Von Mises equivalent stress, $\dot{\varepsilon}_{0}$ is a reference strain rate and $\sigma_{0}^{(r)}$ is the flow stress for each phase $(r)$.

The material parameters used for the simulations are the same as in Lahellec and Suquet (2007b) and Berbenni and Capolungo (2014) with a fibre volume fraction of $f=0.21 . \sigma_{0}^{(M)}=1 \mathrm{GPa}$ for the matrix while $\sigma_{0}^{(I)}=5 \sigma_{0}^{(M)}$ for the inclusions which are considered as hard fibres. The elastic properties, which are assumed to be isotropic, are the same for both phases (Young's modulus $E=100 \mathrm{GPa}$ and Poisson ratio $\nu=0.45$ ). The reference strain rate is set to $\dot{\varepsilon_{0}}=1 \mathrm{~s}^{-1}$ for both phases and the strain rate sensitivity parameter $m$ is either equal to 1 (linear case) or 0.2 (non-linear case). In the SC-TF-AFF, SC-TFSEC and MT-TF-AFF formulations, fibres are modeled with axisymmetric ellipsoidal inclusions elongated in the $x_{3}$ direction and with an aspect ratio of $1 / 100$. The applied strain rate $\dot{E}$ is isochoric and corresponds to in-plane shear loading at $45^{\circ}$ with respect to axis $x_{1}$ :

$$
\dot{E}=\dot{E}_{11}\left(\begin{array}{ccc}
1 & 0 & 0 \\
0 & -1 & 0 \\
0 & 0 & 0
\end{array}\right) \text { with } \dot{E}_{11}=0.5 \mathrm{~s}^{-1}
$$

The calculated overall stress-strain responses obtained from the present SCTF-AFF approach are first compared to the results given by the FFT method (Lahellec and Suquet, 2007b), the SEC (EIV+HS) approach (Lahellec and 


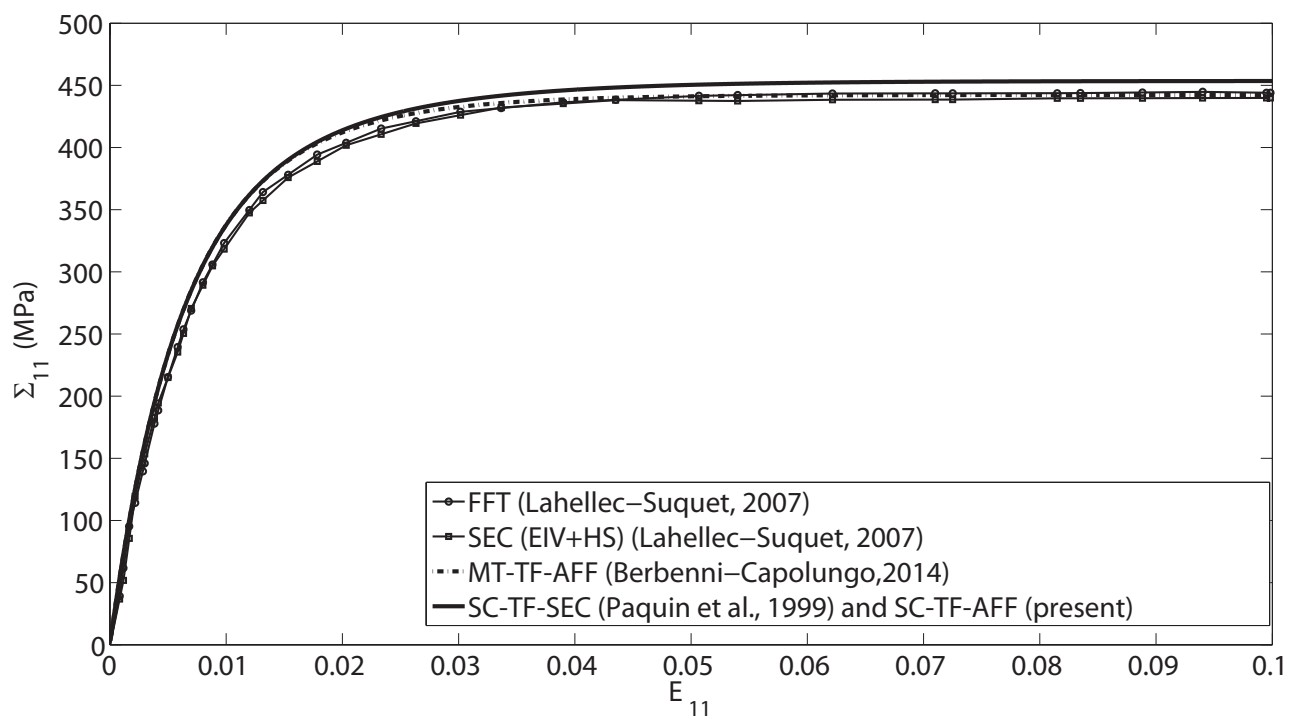

Figure 1. Overall (i.e. effective) monotonic responses obtained from different methods for two-phase elasto-viscoplastic fibre-reinforced composites subjected to inplane shear loading perpendicular to the fibres (fibre volume fraction $f=0.21$, strain rate sensitivity parameter $m=1$ ).

Suquet, 2007b) and the MT-TF-AFF approach (Berbenni and Capolungo, 2014) in figures 1 and 2 for two different strain-rate sensitivities $m=1$ and $m=0.2$, as initially done in Lahellec and Suquet (2007b).

Figure 1 reports the results of the SC-TF-AFF approach for $m=1$ (linear case). In this case, both previous (Paquin et al., 1999) and present translated field approaches are equivalent and lead to the same results. The SC-TF (SEC or AFF) approach gives an overall response that is slightly stiffer than the ones obtained from the FFT method, the SEC (EIV+HS) approach or the MT-TF-AFF approach. In this case, this is clearly due to the self-consistent approximation itself applied to this two-phase microstructure with hard fibres being embedded in a soft matrix phase, for which the HS or the MT estimates classically represent a lower bound of the effective behavior for single-potential materials.

For $m=0.2$ (non-linear case, see figure 2), more discrepancies occur between the different approximations and the FFT method especially at large physical times when the overall behavior tends to the viscoplastic asymptotic state. In this case, all the mean field approximations overestimate the FFT results. In comparison with the linear case, it is still observed that the asymptotic estimate given by the SC-TF-AFF approach is stiffer than the MT-TF-AFF one. However, in agreement with the FFT results, the transient regime determined with the present SC-TF-AFF approach is shorter than for $m=1$. Furthermore, the present SC-TF-AFF approach provides results which are closer to the FFT response than the ones given by the original SC-TF-SEC 


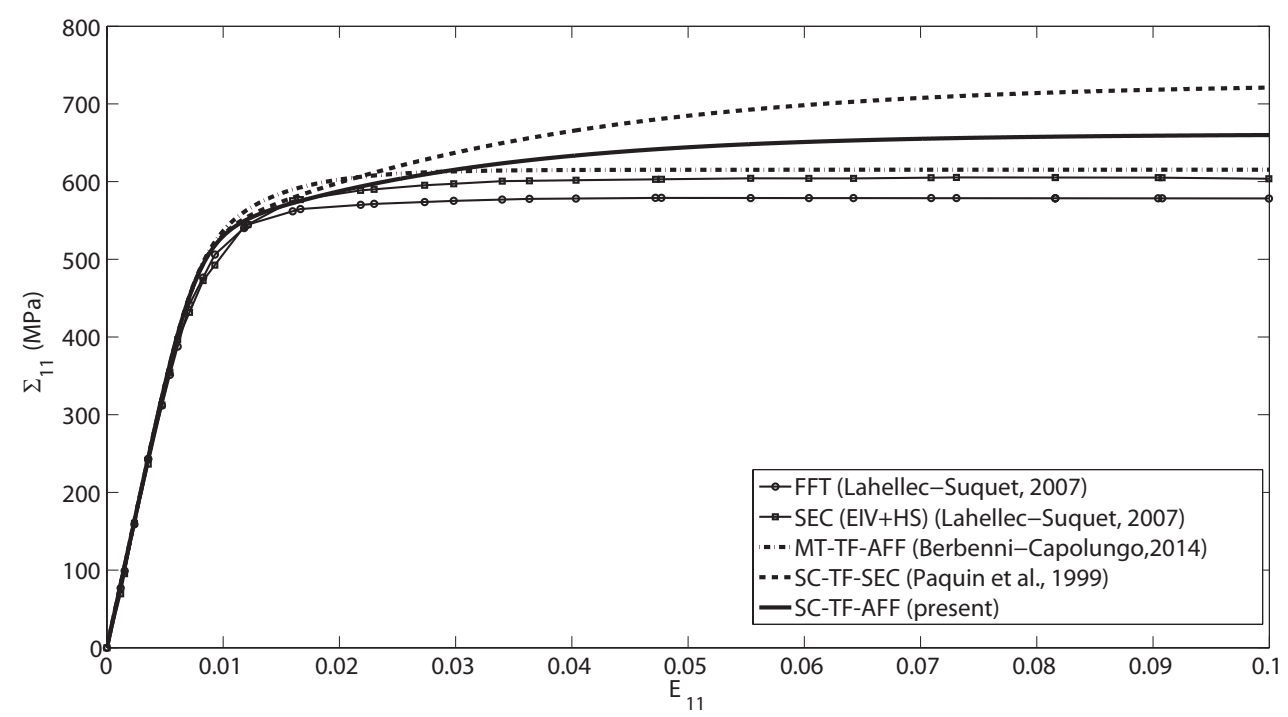

Figure 2. Overall (i.e. effective) monotonic responses obtained from different methods for two-phase elasto-viscoplastic fibre-reinforced composites subjected to inplane shear loading perpendicular to the fibres (fibre volume fraction $f=0.21$, strain rate sensitivity parameter $m=0.2$ ).

method. Also, for such microstructures with a relatively low volume fraction of fibres, these results (figures 1 and 2) confirm that the Mori-Tanaka's estimate, derived by Berbenni and Capolungo (2014), or the Hashin's CCA, developed by Lahellec and Suquet (2007b), give more accurate results than the self-consistent scheme, especially regarding the asymptotic state at large physical times. However, for $m=0.2$, figure 2 shows that the overall response calculated from the SC-TF-AFF approach during the transition from the elastic regime to the viscoplastic regime, i.e. up to $E_{11}=0.03$, is close to the FFT solution.

In figure 3, the phase average stress-strain responses (fibres with dotted lines, matrix with dashed lines) are reported in addition to the overall responses (solid lines) according to both the SC-TF-AFF and SC-TF-SEC approaches. It is observed that the overall stiffness and the average stress (i.e. the first moment of stresses) in the fibres are largely overestimated with the SC-TF-SEC method of Paquin et al. (1999) while they are much reduced with the present SC-TF-AFF formulation. Thus, in comparison with the secant formulation originally developed by Paquin et al. (1999), the description of the mechanical behavior of two-phase materials is significantly improved with the proposed affine formulation. 


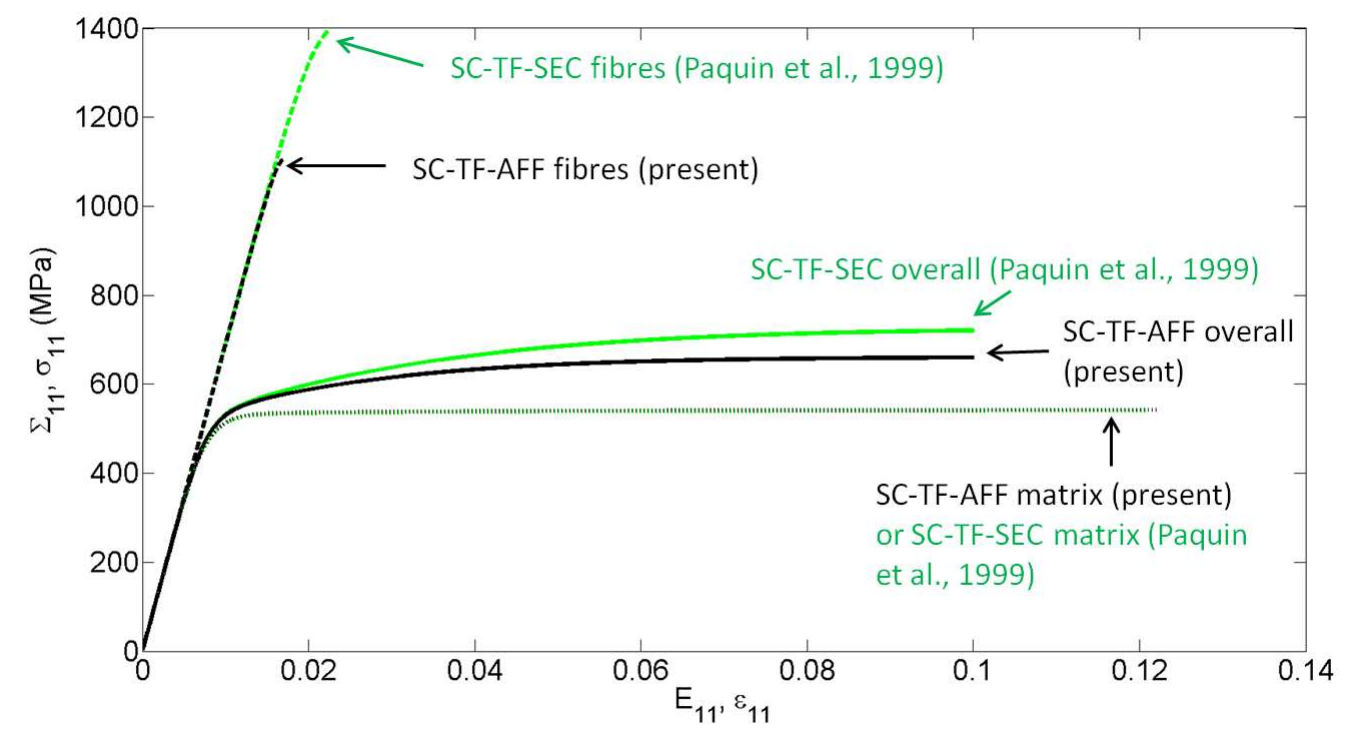

Figure 3. Overall and phase average monotonic responses obtained from the present SC-TF-AFF affine approach and compared to the SC-TF-SEC secant approach (Paquin et al., 1999) for two-phase elasto-viscoplastic fibre-reinforced composites subjected to in-plane shear loading perpendicular to the fibres (fibre volume fraction $f=0.21$, strain rate sensitivity parameter $m=0.2$ ).

\subsection{Polycristalline materials}

In the case of polycrystalline materials, the local behavior of each crystal strongly depends on its orientation because of the anisotropy of plastic, elastic and thermal properties. As a result, the macroscopic behavior is importantly impacted by the internal stresses generated during a loading path because of the interactions between the different grains. In this section, different models are compared for various thermomechanical loading conditions: the proposed affine self-consistent model (labeled as SC-TF-AFF), the original secant selfconsistent model of Paquin et al. (1999) (labeled as SC-TF-SEC) and the FFT-based spectral method (Lebensohn et al., 2012; Mareau et al., 2013; Moulinec and Suquet, 1998) (labeled as FFT). In the present case, the FFTbased spectral method uses the basic algorithm proposed by Moulinec and Suquet (1998) to solve the integral equation associated with the heterogeneous problem. In order to assess the validity of the proposed model, the results of the spectral method are taken as reference since this method provides a numerical solution to the integral equation without neglecting non-local interactions.

The polycrystal, which is used for the comparison, consists of 200 randomly oriented grains with spherical shape. In the case of the FFT-based spectral method, a 3D microstructure was generated using the procedure proposed by Robert et al. (2012). The different grains were initially approximated by spheroids being randomly positioned but not superimposed. The Watershed 
algorithm (Meyer, 1994) was then used to dilate the spheroids to fill the volume element such that smooth grain boundaries were obtained. The microstructure was then discretized into a $128 \times 128 \times 128$ regular grid.

\subsubsection{Single crystal constitutive law}

For the present comparison, the local behavior is described within a crystal plasticity framework. The phenomenological constitutive equations developed by Méric and Cailletaud (1991) are used to model the behavior of each crystal. Considering a crystal with $N$ slip systems, the constitutive law can be summarized in the following set of relations:

$$
\begin{gathered}
\dot{\varepsilon}_{t h}=\alpha \dot{T} \\
\dot{\varepsilon}_{v p}=\sum_{s=1}^{N} \frac{1}{2}\left(m^{s} \otimes b^{s}+b^{s} \otimes m^{s}\right) \dot{\gamma}^{s} \\
\dot{\gamma}^{s}=\left(\frac{\left\langle\left|\tau^{s}-x^{s}\right|-r^{s}\right\rangle}{K^{s}}\right)^{n^{s}} \operatorname{sign}\left(\tau^{s}-x^{s}\right) \\
\tau^{s}=\sigma:\left(b^{s} \otimes m^{s}\right) \\
x^{s}=a^{s} y^{s} \text { with } \dot{y}^{s}=\dot{\gamma}^{s}-d^{s} y^{s}\left|\dot{\gamma}^{s}\right| \\
r^{s}=r_{0}^{s}+\sum_{t=1}^{N} Q H^{s t} q^{t} \text { with } \dot{q}^{t}=\left(1-B q^{t}\right)\left|\dot{\gamma}^{t}\right|
\end{gathered}
$$

In the above equations, $m^{s}$ and $b^{s}$ are respectively the slip plane normal and the slip direction for the $s^{\text {th }}$ slip system. For each slip system, the viscoplastic shear strain rate $\dot{\gamma}^{s}$ depends on:

- the resolved shear stress $\tau^{s}$ which is connected to the stress tensor $\sigma$,

- the isotropic hardening shear stress $r^{s}$ which is the force associated to the internal variable $q^{s}$,

- the kinematic hardening shear stress $x^{s}$ which is the force associated to the internal variable $y^{s}$.

Different material parameters have been introduced in the constitutive model: $\alpha$ is the thermal expansion tensor, $K$ and $n$ are viscosity parameters, $a$ and $d$ are kinematic hardening parameters and $Q, B$ and $H$ are isotropic hardening parameters. $H$ is a $N \times N$ matrix providing a description of the interactions between the different slip systems.

\subsubsection{Uniaxial tension}

First, the case of a polycrystal subjected to uniaxial tension is considered. A constant macroscopic strain rate $\left(\dot{E}_{11}=1 \mathrm{~s}^{-1}\right)$ is imposed in the $x_{1}$ direction 


\begin{tabular}{cccc}
\hline \hline$E(\mathrm{GPa})$ & $\nu$ & $K(\mathrm{MPa})$ & $n$ \\
200 & $1 / 3$ & 200 & 1,5 and 10 \\
\hline \hline
\end{tabular}

Table 1

Material parameters for uniaxial tension simulations. Only non-zero coefficients are indicated. Single crystal elastic properties are assumed to be isotropic ( $E$ is the Young modulus and $\nu$ is the Poisson ratio).

while, except from $\Sigma_{11}$, the macroscopic stress tensor components are set to zero. The plastic flow rule is established for a f.c.c. crystal with slip systems belonging to the $\{111\}<110>$ family. As shown in table 1 , different set of parameters have been used. The set of parameters only differ by the value of the $n$ exponent which varies from 1 to 10 . These different $n$ values allow testing the efficiency of the linearization procedure of the viscoplastic flow rule as a function of non-linearity.

The macroscopic stress-strain curves obtained with the different models and different set of parameters are plotted in figure 4 . In the case of linear viscoelasticity (i.e. $n=1$ ), no difference is observed between the secant and affine formulations since they both yield the same exact definition of the viscoplastic compliances tensor. Also, the results obtained from the self-consistent models are very similar to the reference solution calculated with the spectral method in both the transient and asymptotic regimes. The good description of the transient regime indicates that the self-consistent approximation of the integral equation provides a realistic representation of the interactions associated with the elasto-viscoplastic space-time couplings.

With an increasing non-linearity, both self-consistent models tend to overestimate the polycrystalline aggregate stiffness. However, no matter the value of $n$, the previsions of the proposed affine formulation are always closer to the reference solution. For the specific case of $n=5$, the results can be compared to those obtained from the hereditary approach of Masson and Zaoui (1999) (labeled as SC-HER-AFF). This approach, which is based on the correspondence principle and an affine linearization procedure of the viscoplastic flow rule, requires a complex numerical implementation. Indeed, the solution is obtained from Laplace-Carson transforms whose inversion to the real time space is generally not trivial. Therefore, the proposed model is an interesting alternative solution since it provides very similar results to the hereditary approach of Masson and Zaoui (1999) while the implementation is much easier.

Also, the previsions from the models are very different when the local stress and strain fields are examined. Figure 5 represents the situation of each grain in an equivalent stress-equivalent strain diagram at the end of the tension loading $\left(E_{11}=0.02\right)$ with the different models. It is observed that the dispersion of the stress and strain fields is largely underestimated by both self-consistent models. For high $n$ values, according to the secant formulation, the local strain 

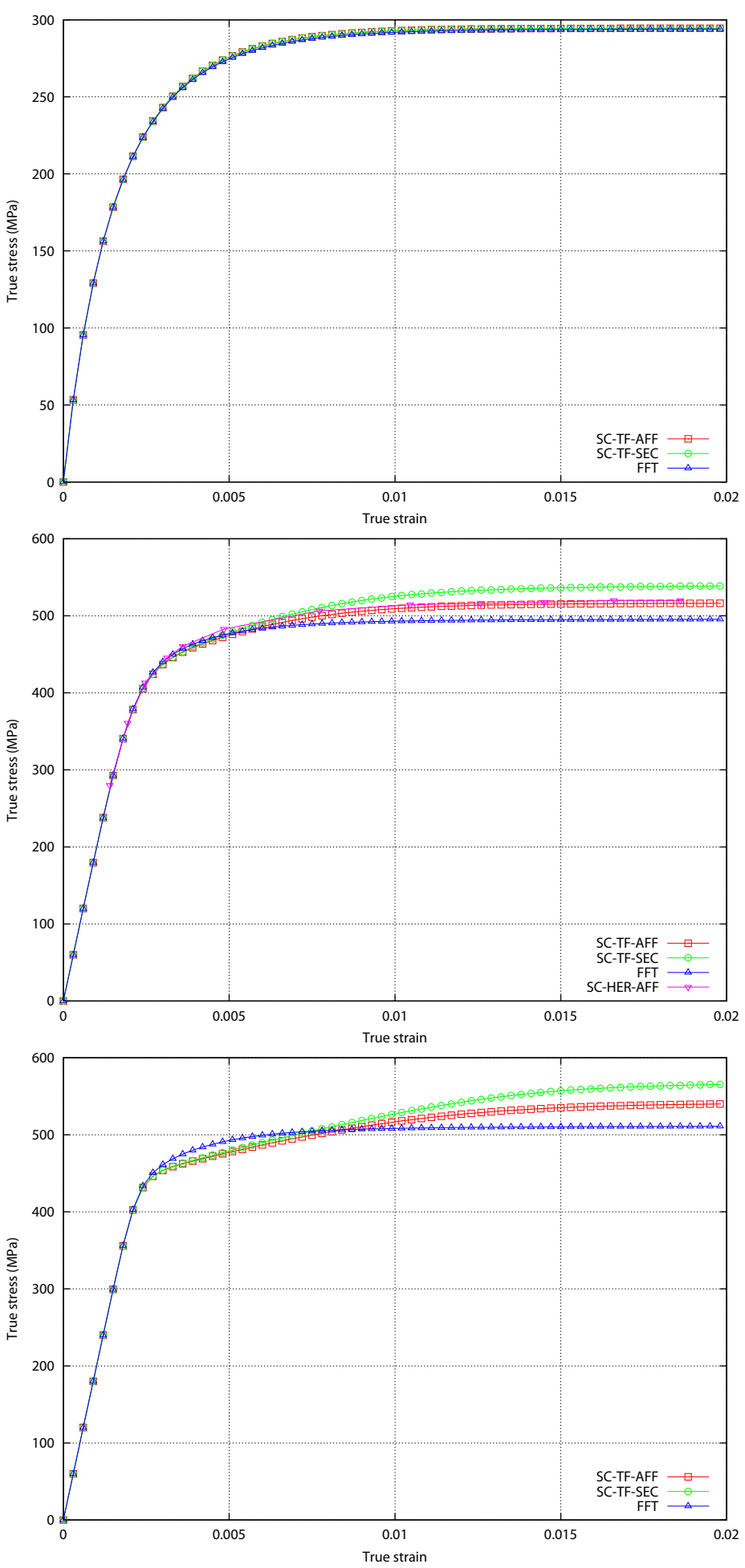

Figure 4. Macroscopic stress-strain curves calculated with the different models for a uniaxial tension test for different $n$ values: $n=1$ (top), $n=5$ (middle) and $n=10$ (bottom). 


\begin{tabular}{cccccc}
\hline \hline$c_{1111}(\mathrm{GPa})$ & $c_{1122}(\mathrm{GPa})$ & $c_{1212}(\mathrm{GPa})$ & $K(\mathrm{MPa})$ & $n$ & \\
197 & 125 & 122 & 12 & 11 & \\
\hline$r_{0}(\mathrm{MPa})$ & $Q(\mathrm{MPa})$ & $B$ & $a(\mathrm{MPa})$ & $d$ & \\
40 & 10 & 3 & 40000 & 1500 & \\
\hline$h_{1}$ & $h_{2}$ & $h_{3}$ & $h_{4}$ & $h_{5}$ & $h_{6}$ \\
1 & 1 & 0.6 & 12.3 & 1.6 & 1.8 \\
\hline \hline
\end{tabular}

Table $\overline{2}$

Material parameters for cyclic plasticity simulations (316L stainless steel) (Guilhem et al., 2013). Only independent elastic constants and non-zero coefficients are indicated.

field is almost equal to the prescribed macroscopic strain. Thus, the secant formulation provides results which are close to the upper bound estimation (i.e. $\varepsilon(x, t)=E(t)$ ) which is obtained from Taylor's model (Taylor, 1938). On the contrary, the affine formulation yields a more realistic distribution of the stress and strain fields though important discrepancies with respect to the reference solution exist. The reason for these differences lies in the description of the influence of neighboring grains. While the spectral method explicitly accounts for neighboring effects as in a N-site modeling (Lebensohn, 2001), the present adopted 1-site self-consistent method describes the interactions between the different grains in an average manner through the properties of the reference homogeneous media. Consequently, on the contrary to the spectral method, self-consistent models do not account for the specific position of each grain.

\subsubsection{Cyclic plasticity}

The case of a 316L stainless steel polycrystalline aggregate subjected to a cyclic loading is now examined. The simulations are performed under strain control with various strain amplitudes $(0.2 \%, 0.4 \%$ and $0.8 \%)$ and with a constant loading frequency $(1 \mathrm{~Hz})$. The material properties, which are given in table 2, have been identified for 316L by Guilhem et al. (2013). Because of the f.c.c. crystal structure, only the $\{111\}<110>$ plastic deformation mode is considered. The interaction matrix $H$ is defined from six different terms $\left(h_{1}\right.$ to $\left.h_{6}\right)$. They correspond to different types of interactions between a given slip system and the other systems. Considering two slip systems ( $s$ and $t$ ), the interaction term $H^{s t}$ depends whether the two considered systems are identical $\left(H^{s t}=h_{1}\right)$, coplanar $\left(H^{s t}=h_{2}\right)$, collinear $\left(H^{s t}=h_{3}\right)$, Hirth junction $\left(H^{s t}=h_{4}\right)$, forming glissile junction $\left(H^{s t}=h_{5}\right)$ or forming sessile junction $\left(H^{s t}=h_{6}\right)$.

The macroscopic responses, which have been computed with the different mod- 

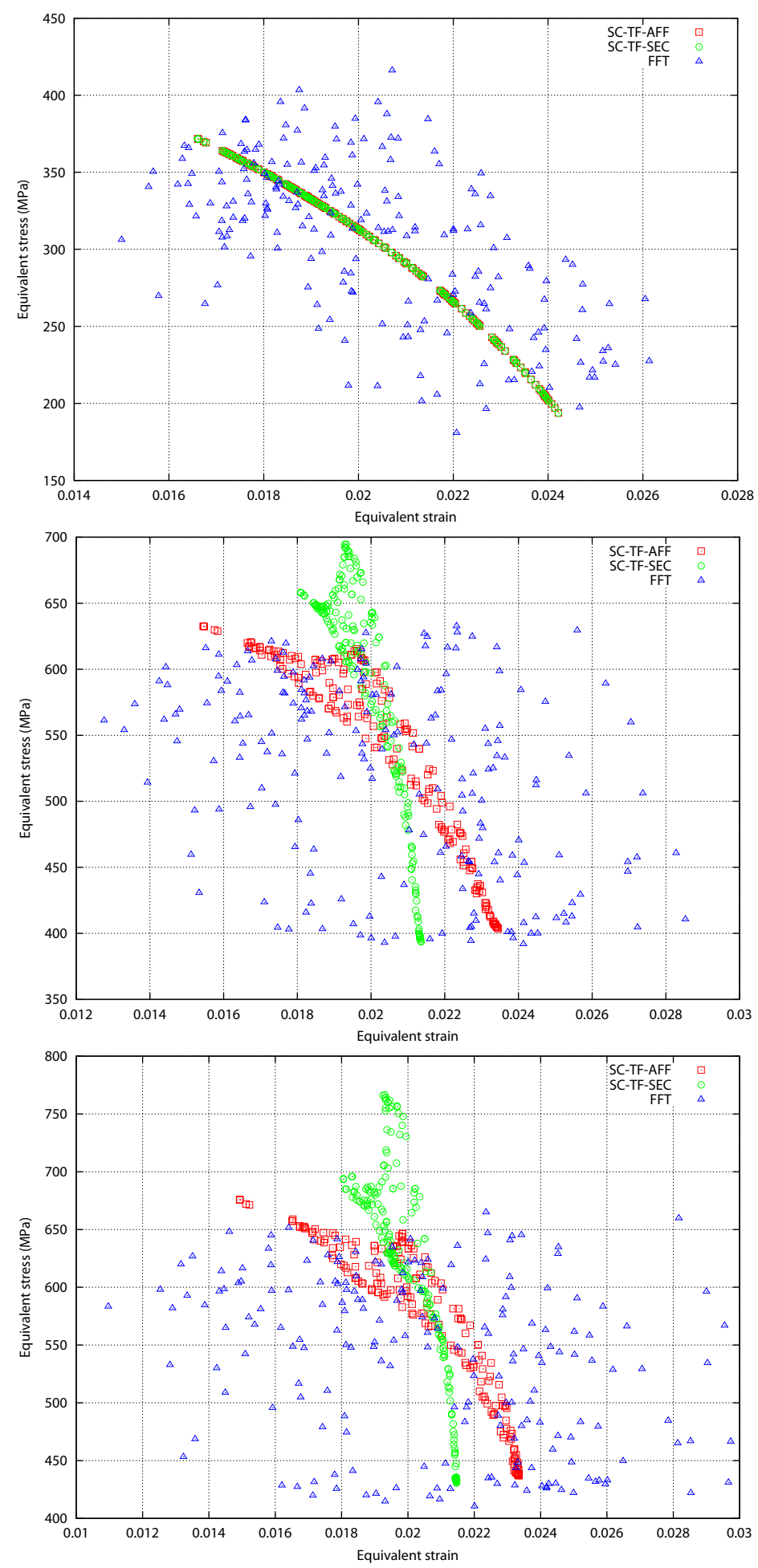

Figure 5. Situation of each grain in an equivalent stress-equivalent strain diagram calculated with the different models at the end of the uniaxial tension simulations ( $E=0.02$ ) for different $n$ values: $n=1$ (top), $n=5$ (middle) and $n=10$ (bottom). 
els, are plotted in figure 6 for the $10^{\text {th }}$ loading cycle. When the strain amplitude is low $(0.2 \%)$, a good agreement is observed between the spectral method and the self-consistent models. However, when the strain amplitude is increased $(0.4 \%$ or $0.8 \%)$, kinematic hardening, which partly results from the development of internal stresses, is largely overestimated by the secant formulation. Indeed, as previously discussed, the secant formulation yields an overestimation of intergranular internal stresses such that kinematic hardening is poorly described with this model. On the contrary, the previsions of the cyclic stressstrain curves determined from the affine model and the spectral method are in very good agreement even for high strain amplitudes.

\subsubsection{Creep}

In order to test the ability of the different formulations to deal with the coupling between thermoelasticity and viscoplasticity within the polycrystalline material, creep simulations are performed. Creep simulations consist of two steps: (i) temperature is first increased to $350^{\circ} \mathrm{C}$ with a rate of $35^{\circ} \mathrm{C} / \mathrm{s}$ then (ii) a constant uniaxial stress (from 300 to $425 \mathrm{MPa}$ ) is applied to the polycrystalline aggregate for $10^{5} \mathrm{~s}$. The single crystal properties which are used for creep simulations were identified for a zirconium alloy (zircaloy-4, h.c.p. crystal structure) at $350^{\circ} \mathrm{C}$ by Diard et al. (2005). They are given in table 3. Four different plastic deformation modes are considered in the simulations: prismatic slip $\{10 \overline{1} 0\}<11 \overline{2} 0>$, basal slip $\{0001\}<11 \overline{2} 0>$, pyramidal $<$ a $>$ slip $\{10 \overline{1} 1\}<11 \overline{2} 0>$ and pyramidal $<$ c + a $>$ slip $\{10 \overline{1} 1\}<11 \overline{2} 3>$.

The strain rate, determined during the secondary creep stage, has been estimated using the different extensions (present affine and secant formulations) and compared to the results from the FFT-based spectral method. The results are reported in figure 7 . For high stress values, the secant formulation underestimates the stationary creep strain rate. This poor prevision of the creep behavior arises from the work hardening associated with the internal stresses which are known to be overestimated by the secant formulation. Thus, it is observed that the thermo-elasto-viscoplastic coupling is better described by the proposed affine formulation for which the agreement with the spectral method is good for any applied stress. This result indicates that the partial relaxation of internal stresses during the second loading step is well captured by the affine model.

Also, the slope of the strain rate versus stress curve corresponds to the effective strain rate sensitivity of the polycrystalline aggregate. While the effective strain rate sensitivity varies smoothly according to the spectral method, abrupt changes are observed with both self-consistent models. These strain rate sensitivity variations can be explained from the activation of the different deformation modes. Indeed, in the case of zircaloy-4, four deformation modes 

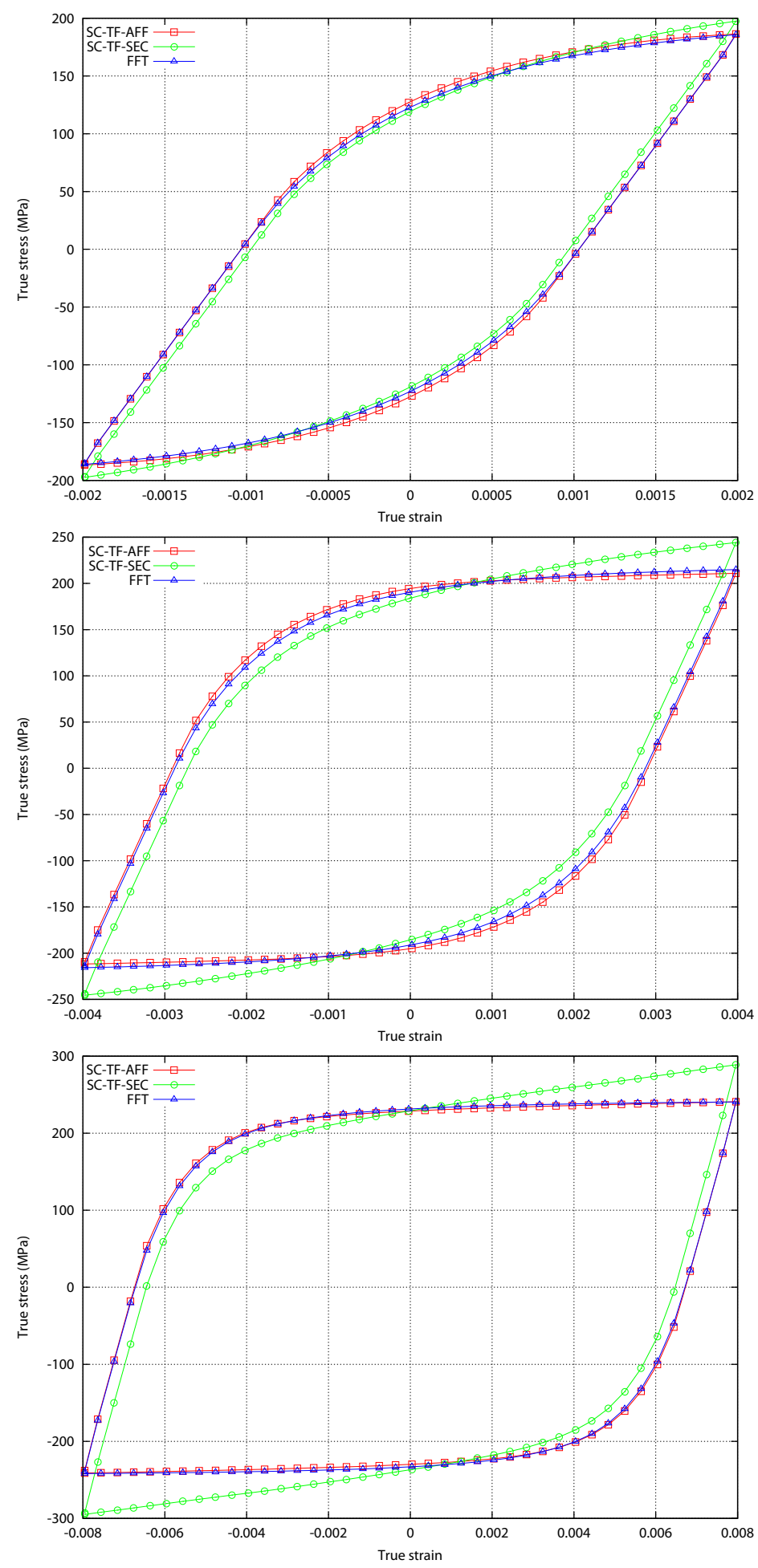

Figure 6. Macroscopic stress-strain curves obtained for a 316L polycrystal subjected to strain-controlled cyclic loadings with different strain amplitudes: $0.2 \%$ (top), $0.4 \%$ (middle) and $0.8 \%$ (bottom). The results correspond to the $10^{\text {th }}$ cycle. 


\begin{tabular}{|c|c|c|c|c|}
\hline$c_{1111}(\mathrm{GPa})$ & $c_{3333}(\mathrm{GPa})$ & $c_{1122}(\mathrm{GPa})$ & $c_{2233}(\mathrm{GPa})$ & $c_{1212}(\mathrm{GPa})$ \\
\hline 103.2 & 118.3 & 51.6 & 46.6 & 23 \\
\hline$\alpha_{11}\left(\mathrm{~K}^{-1}\right)$ & $\alpha_{33}\left(\mathrm{~K}^{-1}\right)$ & & & \\
\hline $5.3 \times 10^{-6}$ & $1.01 \times 10^{-5}$ & & & \\
\hline \multicolumn{5}{|c|}{ Prismatic } \\
\hline$r_{0}(\mathrm{MPa})$ & $K(\mathrm{MPa})$ & $n$ & $a(\mathrm{MPa})$ & $d$ \\
\hline 20 & 198 & 9.4 & 22100 & 170 \\
\hline \multicolumn{5}{|c|}{ Basal } \\
\hline$r_{0}(\mathrm{MPa})$ & $K(\mathrm{MPa})$ & $n$ & & \\
\hline 132 & 90 & 5.5 & & \\
\hline \multicolumn{5}{|c|}{ Pyramidal $<$ a $>$} \\
\hline$r_{0}(\mathrm{MPa})$ & $K(\mathrm{MPa})$ & $n$ & & \\
\hline 107 & 500 & 7.5 & & \\
\hline \multicolumn{5}{|c|}{ Pyramidal $<\mathrm{c}+\mathrm{a}>$} \\
\hline$r_{0}(\mathrm{MPa})$ & $K(\mathrm{MPa})$ & $n$ & $a(\mathrm{MPa})$ & $d$ \\
\hline 195 & 55 & 11.3 & 6120 & 170 \\
\hline
\end{tabular}

Table $\overline{\overline{3}}$

Material parameters for creep simulations (zircaloy-4) (Diard et al., 2005). Only independent elastic constants and non-zero coefficients are indicated.

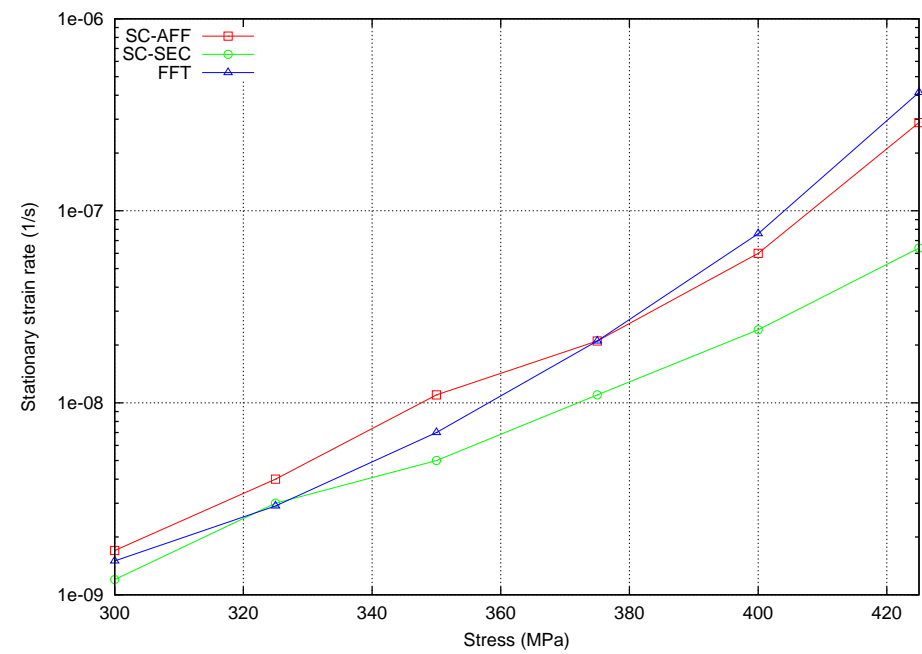

Figure 7. Steady creep strain rate calculated with the different extensions (present affine and secant formulations) and the FFT method for a zircaloy-4 polycrystalline aggregate as a function of the applied stress. 
with different strain rate sensitivities exist. As a result, the evolution of the effective strain rate sensitivity strongly depends on the active deformation modes. According to the FFT method, the activation of a deformation mode in a given grain occurs progressively when the applied stress is increased since intragranular fluctuations of the stress field are accounted for. On the contrary, self-consistent models consider only the volume average strain and stress tensors in each grain to determine the overall behavior. Thus, the activation of a deformation mode takes place simultaneously over the entire grain volume. The abrupt strain rate sensitivity variations being observed at a macroscopic scale with both self-consistent models are therefore caused by the rapid transitions from one deformation mode to another occurring in different grains.

\section{Conclusions}

Translated field techniques were used to build up a new self-consistent model for materials with a local thermo-elasto-viscoplastic behavior. The present homogenization scheme is based on a first order affine linearization of the viscoplastic flow rule inspired from the initial work of Masson et al. (2000). The proposed model can be seen as an extension of the classical secant formulation first developed for elasto-viscoplastic materials by Paquin et al. (1999) without altering the CPU time needed for computations. When compared to reference solutions calculated from a FFT spectral method, a good description of the thermomechanical behavior is obtained with the present model. More specifically, in comparison with the original secant formulation, the description of the interactions between the different phases of a representative volume element is largely improved with the proposed affine model. Furthermore, although the spatial inter-granular elasto-viscoplastic interactions are complex, the present model does not require intensive computations since it is entirely formulated in the real-time space. It is an alternative approach to other recent polycristalline models (e.g. Wang et al., 2010) that should be considered with interest for applications where the computation time is a critical issue, e.g. fatigue, creep responses of polycrystalline materials.

\section{Acknowledgments}

One of the author (S.B.) would like to thank the support of the French State through the National Research Agency (ANR) under the program Investment in the future (Labex DAMAS referenced as ANR-11-LABX-0008-01). 


\section{References}

Berbenni, S., Capolungo, L., 2014. A Mori-Tanaka homogenization scheme for non-linear elasto-viscoplastic heterogeneous materials based on translated fields: an affine extension. Comptes Rendus de Mecanique (special issue), accepted for publication.

Berbenni, S., Favier, V., Lemoine, X., Berveiller, M., 2004. Micromechanical modeling of the elastic-viscoplastic behavior of polycrystalline steels having different microstructures. Materials Science Engineering A 372, 128-136.

Brassard, L., Stainier, L., Doghri, I., Delannay, L., 2012. Homogenization of elasto-(visco)plastic composites based on an incremental variational principle. International Journal of Plasticity 36, 86-112.

Diard, O., Leclercq, S., Rousselier, G., Cailletaud, G., 2005. Evaluation of finite element based analysis of 3D multicrystalline aggregates plasticity: Application to crystal plasticity model identification and the study of stress and strain fields near grain boundaries. International Journal of Plasticity 21, 691-722.

Gilormini, P., 1995. Insuffisance de l'extension classique du modèle autocohérent au comportement non linéaire. Comptes Rendus Academie des Sciences Paris 320, 115-122.

Guilhem, Y., Basseville S., Curtit, F., Stéphan, J.M., Cailletaud, G., 2013. Numerical investigations of the free surface effect in three-dimensional polycrystalline aggregates. Computational Materials Science 70, 150-162.

Harren, S.V., 1991. The finite deformation of rate dependent polycrystals I and II. Journal of the Mechanics and Physics of Solids 39, 345-383.

Hashin, Z., 1969. The inelastic inclusion problem. International Journal of Engineering Science 7, 11-36.

Hershey, A.V., 1954. The elasticity of an isotropic aggregate of anisotropic cubic crystals. ASME, Journal of Applied Mechanics 21, 236-240.

Hill, R., 1965. Continuum micro-mechanics of elastoplastic polycrystals. Journal of the Mechanics and Physics of Solids 13, 89-101.

Hutchinson, J.W., 1976. Bounds and self-consistent estimates for creep of polycrystalline materials. Proceedings of the Royal Society of London A 348, 101-127.

Kröner, E., 1958. Berechnung der elastischen Konstanten des Vielkristalls aus den konstanten des Einskristalls. Zeitschrift fur Physik 151, 504.

Kröner, E., 1961. Zur plastischen Verformung des Vielkristalls. Acta Metallurgica 9, 155-161.

Kröner, E., 1989. Modified green functions in the theory of heterogeneous and/or anisotropic linearly elastic media, in: Weng, G.J., Taya, M., Abe, M. (Eds.), Micromechanics and inhomogeneity, Springer, Berlin, pp. 197211.

Lahellec, N., Suquet, P., 2007. Effective behavior of linear viscoelastic composites: A time-integration approach. International Journal of Solids and Structures 44, 507-529. 
Lahellec, N., Suquet, P., 2007. On the effective behavior of nonlinear inelastic composites: I. Incremental variational principles. Journal of the Mechanics and Physics of Solids 55, 1932-1963.

Lahellec, N., Suquet, P., 2013. Effective response and field statistics in elastoplastic and elasto-viscoplastic composites under radial and non radial loadings. International Journal of Plasticity 42, 1-30.

Laws, N., McLaughlin, R., 1978. Self-consistent estimates for the viscoelastic creep compliances of composite materials. Proceedings of the Royal Society of London A 359, 251-273.

Lebensohn, R.A., Tomé, C.N., 1993. A self-consistent anisotropic approach for the simulation of plastic deformation and texture development of polycrystals: Application to zirconium alloys. Acta Metallurgica et Materialia 41, 2611-2624.

Lebensohn, R.A., 2001. N-site modeling of a 3D viscoplastic polycrystal using Fast Fourier Transform. Acta Materialia 49, 2723-2737.

Lebensohn, R.A., Kanjarla, A.K., Eisenlohr, P., 2012. An elasto-viscoplastic formulation based on fast fourier transforms for the prediction of micromechanical fields in polycrystalline materials. International Journal of Plasticity 32-33, 59-69.

Levin, V.M., 1967. Thermal expansion coefficients of heterogeneous materials. Mekhanika Tverdogo Tela 2, 8894 (English translation: Mechanics of Solids $11,5861)$.

Mareau, C., Cuillerer, D., Morel, F., 2013. Experimental and numerical study of the evolution of stored and dissipated energies in a medium carbon steel under cyclic loading. Mechanics of Materials 60, 93-106.

Masson, R., Zaoui, A., 1999. Self-consistent estimates for the rate-dependent elastoplastic behaviour of polycrystalline materials. Journal of the Mechanics and Physics of Solids 47, 1543-1568.

Masson, R., Bornert, M., Suquet, P., Zaoui, A., 2000. Affine formulation for the prediction of the effective properties of nonlinear composites and polycrystals. Journal of the Mechanics and Physics of Solids 48, 1203-1227.

Mercier, S., Molinari, A., 2009. Homogenization of elasticviscoplastic heterogeneous materials: Self-consistent and Mori-Tanaka schemes. International Journal of Plasticity 25, 1024-1048.

Mercier, S., Molinari, A., Berbenni, S., Berveiller, M., 2012. Comparison of different homogenization approaches for elastic-viscoplastic materials. Modelling and Simulation in Materials Science and Engineering 20, 024004.

Méric, L., Cailletaud, G., 1991. Single crystal modeling for structural calculations: Part 2 - Finite element implementation. ASME, Journal of Engineering Materials and Technology 113, 171-182.

Meyer, F., 1994. Topographic distance and watershed lines. Signal Processing $38,113-125$.

Molinari, A., Canova, G.R., Ahzi, S., 1987. A self consistent approach of the large deformation polycrystal viscoplasticity. Acta Metallurgica 35, 29832994 . 
Molinari, A., Ahzi, S., Kouddane, R., 1997. On the self-consistent modeling of elasticplastic behavior of polycrystals. Mechanics of Materials 26, 43-62.

Molinari, A., 2002. Averaging models for heterogeneous viscoplastic and elastic-viscoplastic materials.Trans. ASME, Journal of Engineering Materials and Technology 124, 62-70.

Moulinec, H., Suquet, P., 1998. A numerical method for computing the overall response of nonlinear composites with complex microstructure. Computer Methods in Applied Mechanics and Engineering 157, 69-94.

Mura, T., 1987. Micromechanics of defects in solids. Kluwer Academic Publishers, Dordrecht, The Netherlands.

Nemat-Nasser, S., Obata, M., 1986. Rate-dependent, finite elasto-plastic deformation of polycrystals. Proceedings of The Royal Society of London A 407, 343-375.

Nemat-Nasser, S., Hori, M., 1994. Micromechanics: Overall Properties of Heterogeneous Materials, North-Holland Series in Applied Mathematics and Mechanics.

Paquin, A., Sabar, H., Berveiller, M., 1999. Integral formulation and selfconsistent modelling of elastoviscoplastic behavior of heterogeneous materials. Archive of Applied Mechanics 69, 14-35.

Pierard, O., Doghri, I., 2006. An enhanced affine formulation and the corresponding numerical algorithms for the mean-field homogenization of elastoviscoplastic composites. International Journal of Plasticity 69, 131-157.

Pierard, O., Llorca, J., Segurado, J., Doghri, I., 2007. Micromechanics of particle-reinforced elasto-viscoplastic composites: Finite element versus affine homogenization. International Journal of Plasticity 23, 1041-1060.

Rekik, A., Auslender, F., Bornert, M., Zaoui, A., 2007. Objective evaluation of linearization procedures in non linear homogenization: a methodology and some implications on the accuracy of micromechanical schemes. International Journal of Solids and Structures 44, 3468-3496.

Robert, C., Saintier, N., Palin-Luc, T., Morel, F., 2012. Micro-mechanical modelling of high cycle fatigue behaviour of metals under multiaxial loads. Mechanics of Materials 55, 112-129.

Rougier, Y., Stolz, C., Zaoui, A., 1994. Self-consistent modelling of elasticviscoplastic polycrystals. Comptes Rendus Academie des Sciences Paris 318, $145-51$.

Sabar, H., Berveiller, M., Favier, V., Berbenni, S., 2002. A new class of micromacro models for elasticviscoplastic heterogeneous materials. International Journal of Solids and Structures 39, 3257-3276.

Taylor, G.I., 1938. Plastic strain in metals. Journal Institute of Metals 61, 307-324.

Wang, H., Wu, P.D., Tomé, C.N., Huang, Y., 2010. A finite strain elasticviscoplastic self-consistent model for polycrystalline materials. Journal of the Mechanics and Physics of Solids 58, 594-612.

Weng, G.J., 1981. Self-consistent determination of time-dependent behavior of metals. ASME, Journal of Applied Mechanics 48, 41-46. 
Weng, G.J., 1981. A self-consistent scheme for the relaxation behaviour of metals. ASME, Journal of Applied Mechanics 48, 779-784.

Zaoui, A., Raphanel, J.L., 1993. On the nature of the intergranular accommodation in the modeling of elastoviscoplastic behavior of polycrystalline aggregates, in: Teodosiu, C. et al (Eds.), Mecamat 91: Large Plastic Deformations, Fundamentals and Application to Metal Forming, Rotterdam, pp. $185-92$. 